\title{
IMPACTO ECONÓMICO DE LAS HELADAS Y DEL FINANCIAMIENTO DE LA RESIEMBRA EN SINALOA
}

\author{
ECONOMIC IMPACT OF FROST AND FINANCING \\ OF REPLANTING IN SINALOA
}

\section{Edgardo Ayala Gaytán Instituto Tecnológico y de Estudios Superiores \\ de Monterrey, México. Campus Monterrey \\ edgardo@itsm.mx}

Joana Chapa Cantú

Universidad Autónoma de Nuevo

León, México

joana.chapacn@uanl.edu.mx

\section{RESUMEN}

Se calculan los efectos de corto plazo de las heladas de febrero de 2011 sobre la economía de Sinaloa. Estimamos el impacto inicial en el sector agrícola mediante técnicas econométricas, y después, lo introducimos dentro de un modelo de equilibrio general de corto plazo para la economía de Sinaloa. Tal que obtenemos los efectos sobre la actividad económica de los sectores productivos, y el ingreso y consumo de los hogares. Finalmente, se evalúan los efectos económicos de dos políticas públicas para contrarrestar el shock climático: resiembra de maíz financiada con recursos federales y resiembra de maíz financiada con recursos estatales.

Palabras clave: modelo de equilibrio general de corto plazo, ayuda por desastre natural, clima.

Clasificación JEL: C69, H84, Q54. 


\begin{abstract}
We calculate the economic damages produced by the February 2011 frost in the northwest state of Sinaloa in Mexico. We estimated the initial impact on the agricultural sector employing time series techniques, and then we introduced such estimation into a short run general equilibrium model for the Sinaloa economy. We obtained the effects on the economic activity of the main sectors, personal income and households' consumption. Finally, the economic effects of two public policies to deal with the climate shock are evaluated: corn replanting financed with federal government's resources and corn replanting financed with state's resources.
\end{abstract}

Keywords: short run general equilibrium model, disaster aid and climate.

* Agradecemos la asistencia técnica de Imelda Abigail Miranda Aquino, así como a los dos árbitros anónimos por sus valiosos comentarios y sugerencias. 


\section{INTRODUCCIÓN}

El 3 y 4 de febrero de 2011 las heladas que afectaron al norte del país provocaron un daño tan importante en el sector agrícola del estado de Sinaloa que se perdió la producción del principal cultivo del estado, el maíz. Esta contingencia climática se esperaba que tuviera serias repercusiones de índole económica, toda vez que la agricultura tiene un peso considerable en la actividad productiva $\mathrm{y}$, con ello, en la generación de ingreso del estado. Ante dicha problemática el gobierno federal apoyó a Sinaloa proporcionando maíz para resembrar 300000 hectáreas dañadas, y así alcanzar una producción de tres millones de toneladas. Una resiembra exitosa, implicaba que sólo se perdería $40 \%$ de la cosecha.

Los efectos de contingencias climáticas han sido analizados internacionalmente con técnicas multisectoriales, ya que permiten cuantificar sus efectos al considerar la interrelación entre los sectores económicos (modelo insumo-producto) y, en su forma más amplia, entre todos los agentes que participan en una economía: hogares, actividades productivas, gobierno y sector externo (modelo de equilibrio general computable). Para el caso de México sólo tenemos conocimiento del trabajo de Boyd e Ibarrarán (2011), quienes analizan los impactos del cambio climático y de políticas de mitigación con un modelo de equilibrio general.

En este contexto, la presente investigación tiene tres objetivos. Primero, estimar el impacto económico de la pérdida de la producción de maíz ocasionada por las heladas de febrero de 2011 sobre la producción agrícola; producto interno bruto estatal, total y por sector; desempleo y variables relacionadas con el bienestar de las familias: ingreso y consumo. Segundo, evaluar en qué grado la resiembra de maíz con la cual el gobierno federal apoyó al estado de Sinaloa ayudó a mitigar los efectos económicos de las heladas. Tercero, determinar los efectos económicos de la resiembra si hubiera sido financiada con recursos estatales, al asumirse una política de redirección del gasto, es decir, que el gobierno de Sinaloa redujera el gasto en bienes y servicios para destinarlo a comprar maíz y resembrar.

Para lograr tales objetivos se estimaron modelos econométricos para dimensionar el impacto inicial de la contingencia climatológica sobre la 
producción agrícola, el PIB primario, el PIB total y la tasa de desempleo del estado de Sinaloa, con y sin resiembra. Además, se especificó un modelo de equilibrio general de corto plazo para Sinaloa, que hace referencia al año 2003, para poder cuantificar el impacto sobre el consumo y el ingreso de los hogares, los niveles de actividad de los sectores económicos y evaluar los efectos de las políticas de resiembra financiadas con recursos de diferentes niveles de gobierno.

En este sentido, el presente trabajo contribuye al análisis de los efectos de contingencias climáticas, en cuanto a combinar técnicas econométricas y modelos multisectoriales para cuantificar los efectos a través del sistema económico del choque y de las políticas públicas para mitigarlo.

El contenido de la investigación es el siguiente. El segundo apartado describe antecedentes de investigaciones realizadas a nivel internacional sobre los efectos de contingencias climáticas. En el tercero se analiza la estructura y dinámica económica de Sinaloa, se discuten los resultados de las estimaciones econométricas de las relaciones entre sus principales variables agregadas y las relacionadas con la agricultura: PIB primario y la producción de los diez principales cultivos del estado, entre ellos el maíz; PIB total y PIB primario y tasa de desempleo y tasa de crecimiento del PIB total. El siguiente apartado contiene los resultados de las simulaciones realizadas con las ecuaciones econométricas y el modelo de equilibrio general de corto plazo para el estado de Sinaloa. El quinto apartado considera las principales conclusiones del trabajo. Se finaliza con dos anexos metodológicos, en el anexo A se discuten las estimaciones econométricas y el anexo B contiene la especificación y calibración del modelo de equilibrio general de corto plazo.

\section{ANTECEDENTES BIBLIOGRÁFICOS}

Los desastres naturales de índole meteorológica, como las sequías o heladas, se traducen en pérdidas económicas importantes, principalmente en el sector agrícola. Por ello, los modelos multisectoriales son de los métodos más utilizados para estudiar sus efectos, ya que permiten analizar y cuantificar el impacto de la contingencia climática al considerar la interdependencia 
entre los agentes económicos. Según su grado de endogeneidad dichos modelos se clasifican en tres tipos: modelos insumo-producto (MIO), modelos de multiplicadores contables (MMC) y modelos de equilibrio general computable (MEGC). ${ }^{1}$

Los modelos insumo-producto permiten determinar los efectos económicos sobre todas las actividades productivas, al considerar la interrelación que existe entre ellas. Los modelos de multiplicadores contables agregan la interrelación existente entre las actividades productivas y los hogares, es decir, el efecto inducido de la relación entre la generación de ingreso desde el sector productivo, cómo este se distribuye hacia los hogares y cómo estos últimos lo gastan en bienes y servicios que proveen los sectores económicos. Los modelos de equilibrio general computable son los más elaborados, puesto que establecen funciones de comportamiento interconectadas para cada uno de los agentes que participan en una economía, empresas o sectores, familias, gobierno y sector externo, por lo que son una herramienta sumamente útil para analizar los efectos no sólo de choques exógenos y de políticas públicas, sino de combinaciones de las mismas.

Dentro de los autores que han utilizado los modelos insumo producto podemos mencionar a Diersen, Taylor y May (2002), quienes estiman pérdidas económicas de 1.8 billones de dólares debido a la sequía de 2002 en Dakota del Sur. Sin embargo, más tarde Diersen y Taylor (2003) al tomar en cuenta las mejores condiciones de mercado y la ayuda directa por parte del gobierno federal ajustaron dichas pérdidas a 1.4 billones de dólares. A nivel país podemos mencionar el trabajo de Wheaton et al. (2008), en donde, utilizando un modelo similar, se estima los costos económicos causados por las sequía de 2001 y 2002 en Canadá, enfocándose principalmente en el sector agrícola.

Por otro lado, el trabajo de Horridge, Madden y Wittwer (2005) constituye una referencia en el uso del MEGC. En dicho estudio se estiman los impactos de las sequías de 2002 y 2003 en Australia. A pesar de la baja participación de la agricultura (3.6\%) en el Producto Interno Bruto (PIB) se

\footnotetext{
${ }^{1}$ Los artículos seminales de este tipo de modelos son: Leontief (1965) en el caso del modelo insumo-producto, Stone (1985) y Pyatt y Round (1979) para el modelo de multiplicadores contables y Shoven y Walley $(1972,1973)$ para el modelo de equilibrio general computable.
} 
estima una disminución del mismo de $1.6 \%$, de la cual 1.0\% se debe a los efectos negativos en el sector de la agricultura y el $0.6 \%$ restante a efectos multiplicadores negativos.

En esta misma línea de estudio, Hodges y Haydu (2003) llevan a cabo un análisis de los impactos económicos de la sequía del año 2000 para la industria de la horticultura de Florida, y mediante modelos económicos regionales determinan el efecto multiplicador en el ingreso. Los resultados muestran que algunos sectores, como los comerciantes y minoristas, se beneficiaron de la sequía.

Para el caso de México, si bien la literatura empírica respecto al tema es escasa, podemos mencionar el caso de Boyd e Ibarrarán (2011), quienes analizan los impactos del cambio climático en México en un marco de equilibrio general. Esto les permite determinar el costo inherente del cambio climático, así como los costos y beneficios de la adopción de políticas de mitigación y adaptación, tanto a nivel agregado como sectorial. En particular, modelan los impactos esperados en hidrología, agricultura, sector forestal, sector industrial y energético; asi como el impacto macroeconómico y distributivo atribuible al cambio climático. En cuanto a los escenarios simulados, la estimación del costo del cambio climático es de $1.1 \%$ del PIB anual. Además, otra aportación importante es la regresividad de este costo, donde los pobres pierden mayor bienestar que los ricos. Los sectores productivos más afectados son la agricultura en más de 11\%, la ganadería en $10 \%$ y el sector forestal en 15 por ciento.

\section{ESTRUCTURA Y DINÁMICA ECONÓMICA DEL ESTADO DE SINALOA}

\subsection{Importancia del sector agrícola en Sinaloa}

La vocación del estado de Sinaloa es claramente agrícola. Se siembran alrededor de 925 mil hectáreas cada año, casi $60 \%$ de ellas de maíz y alrededor de $90 \%$ entre maíz, sorgo y frijol. La producción de los principales diez cultivos ascendió a 23.7 mil millones de pesos en el año 2009, es decir, una densidad económica de poco más de 25 mil pesos por hectárea. 
Como se puede apreciar en la gráfica 1, el principal cultivo de acuerdo con su aportación a la producción, en pesos corrientes, es el maíz, con aproximadamente $60 \%$, seguido del tomate rojo con $13 \%$, el frijol $10.1 \%$, y el chile verde con $8.4 \%$, los cuales, en conjunto, representan 91.5 por ciento.

\section{Gráfica 1}

Distribución del valor de la producción agrícola en Sinaloa (2009)

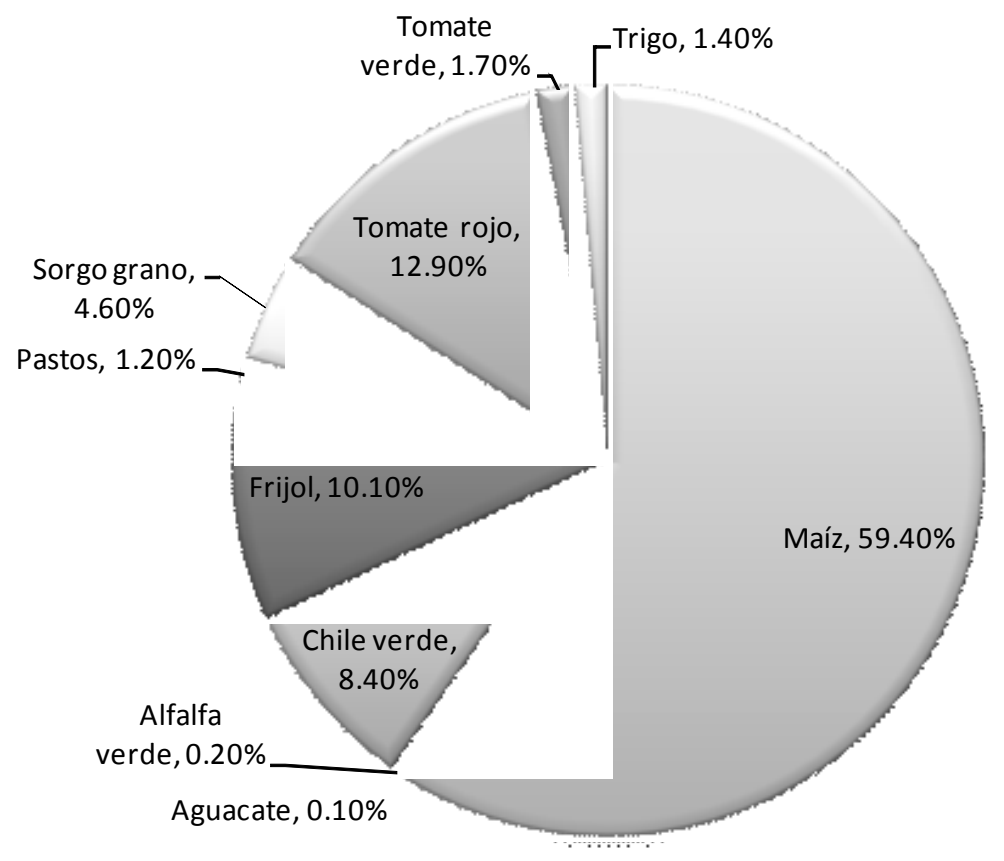

Fuente: Secretaría de Agricultura, Ganadería, Desarrollo Rural, Pesca y Alimentación (SAGARPA).

La vocación agrícola de Sinaloa se puede apreciar por el peso que representan los distintos sectores en su economía. En 2009 el sector primario (que incluye agricultura, ganadería, silvicultura y pesca) representó $13.1 \%$ del PIB total del estado, el secundario (manufactura) $17.7 \%$ y el terciario (servicios) el restante $71.1 \%$. En cambio, en el ámbito nacional, 
el sector primario representa tan sólo 4\%, es decir, pesa más de 3 veces para la economía de Sinaloa de lo que representa para todo el país. Más formalmente podemos estimar el índice de ventaja comparativa revelada o ventaja comparativa de Balassa (CVR), el CVR permite conocer los sectores donde un país o región tiene ventaja competitiva y, por lo tanto, donde tiene potencial exportador. El CVR del sector $i$ se obtiene al calcular el cociente de la participación de las exportaciones del sector $i$ de Sinaloa en las exportaciones totales de Sinaloa y de la participación de las exportaciones del sector $i$ de toda la nación en las exportaciones totales nacionales. Índices iguales o menores a la unidad indican ausencia de ventaja comparativa, en cambio índices mayores a la unidad señalan ventaja comparativa y, por ello, capacidad exportadora.

En promedio, durante el periodo 2003-2009 el CVR del sector primario promedió 3.28, mientras que para el sector secundario fue de sólo 0.55 y de 1 para el terciario. Claramente, la ventaja comparativa de Sinaloa son las actividades primarias. ${ }^{2}$

\subsection{Dinámica del sector de la agricultura, ganadería, caza y pesca en Sinaloa}

¿Qué explica la dinámica de la producción interna bruta del sector de agricultura, ganadería, silvicultura, caza y pesca (PIB primario)? La gráfica 2 muestra la evolución reciente del valor de la producción de los diez cultivos más importantes de Sinaloa, a pesos del 2003, y el producto interno bruto de la agricultura, ganadería, silvicultura y pesca del estado a precios constantes. El alto grado de correlación es evidente, es decir, se trata de una imagen espejo, al parecer cuando se incrementa o disminuye la producción agrícola también lo hace en el mismo sentido el PIB primario. No es extraño, por lo tanto, asumir que la dinámica del sector primario de Sinaloa está estrechamente ligada al valor de la producción de los diez principales cultivos del estado: maíz, sorgo, trigo, frijol, alfalfa, chile verde, aguacate, pastos, tomate rojo y verde.

\footnotetext{
${ }^{2}$ Este índice se define y calcula con base en las exportaciones, variable económica que desafortunadamente en México no contamos con ella, ni en el entorno regional, ni sectorial. Por tal motivo, utilizaremos el producto interno bruto como indicador del nivel de exportaciones por sector y región.
} 


\section{Gráfica 2}

Valor de la producción y del PIB primario (agricultura, ganadería, silvicultura, caza y pesca) a pesos del 2003 en Sinaloa

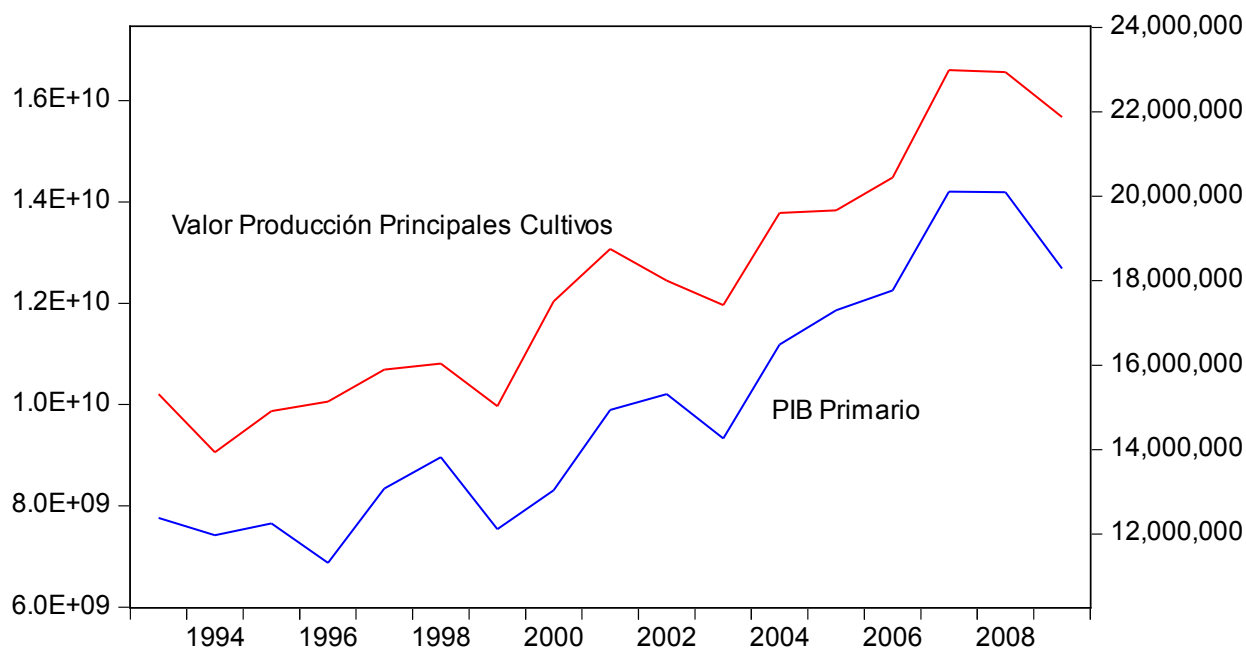

Fuente: SAGARPA e Instituto Nacional de Estadística, Geografía e Informática (INEGI).

Mediante la estimación econométrica de la relación entre el PIB primario, es decir, del PIB de la agricultura, ganadería, sivilcultura, caza y pesca y la producción de los diez principales cultivos de Sinaloa, se pudo determinar que un cambio de $10 \%$ en el valor de la producción de los principales cultivos se transfiere en $6.54 \%$ de crecimiento al PIB primario del estado. ${ }^{3}$

\subsection{Agricultura y la dinámica económica de Sinaloa}

Ahora, la pregunta es: ¿cómo afecta el valor de la producción de los principales cultivos a la economía del estado, medida por el PIB total de Sinaloa? Una primera aproximación es la de usar el peso relativo del sector primario en la economía estatal, que, como vimos, es de aproximadamente

\footnotetext{
${ }^{3}$ Los detalles de la estimación están contenidos en el anexo metodológico A.1.
} 
13 por ciento. Sin embargo, el cálculo es incorrecto, toda vez que asume que no hay efectos indirectos sobre los otros sectores, principalmente el terciario (servicios), esto es claramente muy restrictivo.

Es decir, la agricultura es una variable crítica de la dinámica de todos los sectores del estado, cuando crece o decrece no sólo cambia el PIB de las actividades primarias, sino que se desatan efectos multiplicadores sobre los sectores de manufacturas y servicios del estado.

La gráfica 3 presenta claramente la asociación positiva entre el PIB primario y el PIB total del estado, la pendiente de la línea de regresión que se traza en este diagrama de dispersión de puntos sugiere que el coeficiente de esta recta es mayor a lo que se pensaría con base en el peso del sector en la economía estatal.

\section{Gráfica 3}

Relación entre el PIB total y primario a pesos de 2003 en Sinaloa*

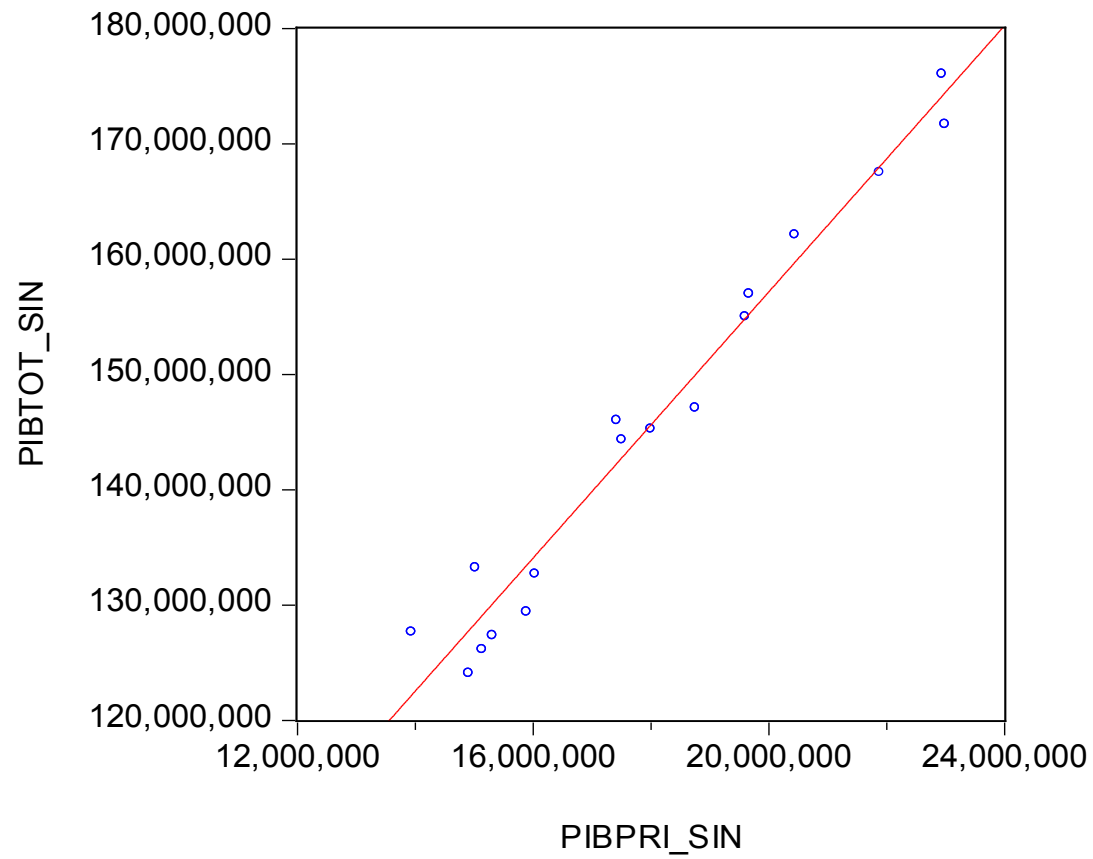

Fuente: elaboración propia con base en datos de la SAGARPA e INEGI.

Notas: *PIBTOT_SIN: producto interno bruto total de Sinaloa. PIBPRI_SIN: producto interno bruto primario (agricultura, ganadería, silvicultura, caza y pesca) de Sinaloa. 
Por tal motivo, decidimos estimar un modelo econométrico que considera al PIB primario y al PIB nacional como determinantes del PIB total de Sinaloa. Es decir, nuestro modelo asume que el estado de Sinaloa está determinado por la dinámica agrícola, sector donde demostramos que el estado tiene clara ventaja competitiva, y la dinámica del país. ${ }^{4}$

Los resultados indican que un incremento de $10 \%$ en el PIB primario se traduce en un aumento en la tasa de crecimiento del PIB total de Sinaloa de 3 por ciento. La magnitud es poco más del doble de la participación de este sector en la economía estatal (13\%). Esto es, tal y como lo comentamos antes, al crecer el sector primario la economía de Sinaloa crece por dos vías: la primera por el efecto directo de la agricultura en el $\mathrm{PIB}$, la segunda por los efectos multiplicadores en la producción de los otros sectores animados por la expansión de ingresos que ocasiona la expansión agrícola. En otras palabras, si usáramos sólo la participación del sector en la economía para hacer el pronóstico de su efecto en la economía total, estaríamos claramente subestimando el efecto.

\subsection{El desempleo y la dinámica económica de Sinaloa}

En Estados Unidos se acuñó el término de la Ley de Okun a la sólida regularidad empírica de la relación entre la tasa de desempleo y el crecimiento económico. ${ }^{5}$ Motivados por este hecho, se decidió probar una especie de Ley de Okun modificada para el caso de Sinaloa, se estima un modelo econométrico que relaciona el cambio en la tasa de desempleo en la entidad y la tasa de crecimiento del PIB estatal. ${ }^{6}$

La relación indica que, si aumenta la tasa de crecimiento en la economía de Sinaloa, la tasa de desempleo disminuye, más concretamente por cada $10 \%$ adicional de crecimiento en el estado, la tasa de desempleo se reduce en casi una quinta parte, esto es, 1.7 por ciento.

\footnotetext{
${ }^{4}$ Los detalles de la estimación están contenidos en el anexo metodológico A.2.

${ }^{5}$ La Ley de Okun, propuesta por el economista norteamericano Arthur Okun, establece la correlación existente entre los cambios en la tasa de desempleo y los cambios en el PIB actual (real). Una vez que se estima esta relación puede establecerse cuánto cambia el desempleo de un país o región por cada punto porcentual que crece el PIB del mismo.

${ }^{6}$ Los detalles de la estimación están contenidos en el apéndice metodológico A.3.
} 


\section{EFECTOS ECONÓMICOS DE LAS HELADAS EN SINALOA}

La helada ocasionó una pérdida de aproximadamente $70 \%$ de las hectáreas sembradas de los cinco cultivos más importantes del estado de Sinaloa, el maíz fue el más afectado. No obstante, al resembrarse al menos 300000 hectáreas de maíz y alcanzar una producción de tres millones de toneladas de este grano básico, el daño se reduciría a $40 \%$ de pérdida en la producción, puesto que se tenía programada una producción de 5 millones en 2011. Esta es la principal afectación de la helada a la agricultura de Sinaloa, porque, como ya mencionamos en la primera sección, el maíz representa $60 \%$ de las hectáreas empleadas en la agricultura del estado y del valor de la producción agrícola total. Con base en la anterior información, realizamos las siguientes simulaciones.

\subsection{Simulaciones}

Simulación 1, situación sin resiembra. Se pierde en su totalidad la producción de maíz del estado de Sinaloa.

Simulación 2, resiembra financiada por el gobierno federal. El plan de resiembra del maíz del gobierno federal tiene éxito, de forma que sólo se pierden dos millones de toneladas, es decir, $40 \%$ de la cosecha de ese grano en el año 2011.

Simulación 3, resiembra financiada por el gobierno estatal. El plan de resiembra del maíz tiene éxito, de manera que solamente se pierden dos millones de toneladas, es decir, 40\% de la cosecha de ese grano en el 2011, pero esta resiembra es financiada por el gobierno estatal.

Cabe comentar, que en el caso de los modelos econométricos, las simulaciones 2 y 3 dan los mismos resultados, ya que el financiamiento de la resiembra del maíz no se considera como variable dentro de ellos. 


\subsection{Resultados}

\subsubsection{Predicciones De Los Modelos ECONOMÉTRICOS}

Para hacer las simulaciones de los efectos de las heladas procedimos de la siguiente forma. primero multiplicamos la cantidad de hectáreas siniestradas por la productividad de las hectáreas en el 2009, para obtener una medida de la reducción de la producción en toneladas de maíz. ${ }^{7}$ Enseguida, la caída resultante en producción se multiplicó por el precio medio rural de 2003 para dicho grano, así obtuvimos la pérdida del valor de la producción agrícola a precios de 2003. Después se calculó la pérdida porcentual al dividir entre el valor de la producción agrícola a precios de 2003 en los ciclos inmediatos anteriores. De tal forma, se obtiene el efecto de las heladas sobre el valor de la producción de maíz bajo los tres escenarios antes descritos (se aplica el mismo procedimiento para el efecto del daño sobre otros cultivos que involucra el escenario medio).

Los resultados de los modelos econométricos comentados en el apartado anterior nos permitirán obtener el impacto de las heladas sobre: PIB primario (agricultura, ganadería, silvicultura, caza y pesca) y PIB total y tasa de desempleo; ya que de estos modelos se desprenden estimaciones de la sensibilidad del: $i$ ) PIB primario con respecto a la producción de los principales cultivos del estado; ii) PIB total del estado con respecto al PIB primario y iii) tasa de desempleo con respecto al crecimiento económico del estado. Como se puede observar, las variables antes mencionadas están ligadas unas con otras mediante los modelos econométricos estimados, de acuerdo con la lógica de la figura $1 .^{8}$

\footnotetext{
${ }^{7}$ En el momento en que se realizaron las estimaciones econométricas de esta investigación, datos requeridos en ellas como el PIB total y el PIB primario de Sinaloa sólo estaban disponibles hasta el año 2009, por ello, y para ser consistente en el uso de la información, en los cálculos descritos en este párrafo se utilizó la productividad por hectárea del año 2009. Cabe decir, que los resultados cambiarían sensiblemente si se utilizara la productividad del año 2010, ya que está fue de 9.96 comparado con 9.76 de 2009.

${ }^{8}$ Con la disminución en el valor de la producción agrícola a precios constantes insertamos el valor en la ecuación (1a) del apéndice metodológico A.1 y asumimos que el residual toma su valor esperado de cero, para obtener un pronóstico para el logaritmo del PIB primario del estado. Ya con una estimación del PIB primario y al asumir que la economía nacional
} 


\section{Figura 1}

Conexiones entre las ecuaciones estimadas de los agregados económicos de Sinaloa

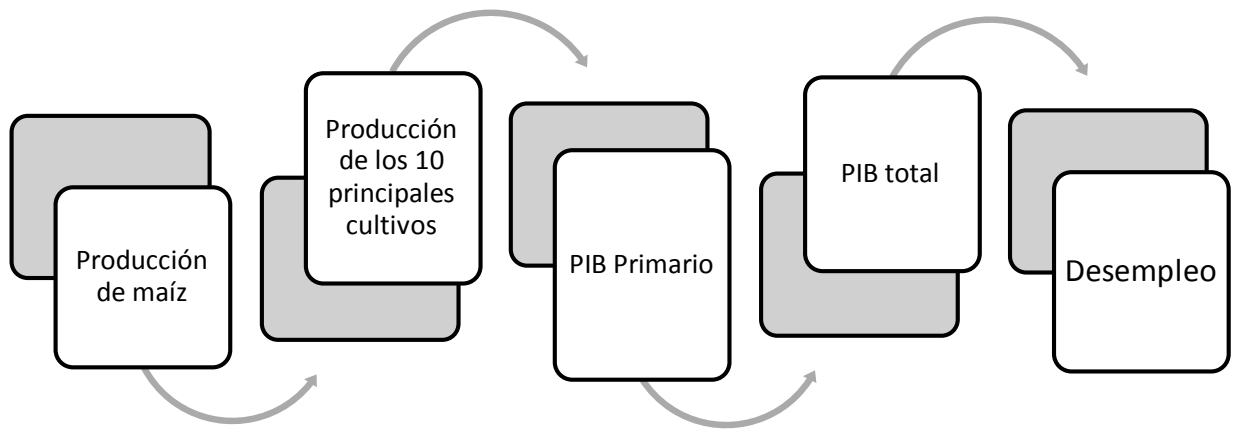

Fuente: elaboración propia.

\section{Simulación 1: Sin resiembra del maíz}

La pérdida total de la producción de maíz en el estado de Sinaloa genera una caída en la producción agrícola de $42.8 \%$, lo cual se traduce en una reducción del PIB primario de $28.0 \%$ y del PIB total de $6.4 \%$. La tasa de desempleo se incrementa a 5.7 por ciento.

\section{Simulación 2 y 3: Con resiembra del maiz}

Independientemente del nivel de gobierno que financie la resiembra de $60 \%$ del maíz siniestrado, se pierde $23 \%$ de la producción agrícola del estado, lo que impacta al PIB primario en $15.1 \%$ y al PIB estatal en 2.5 por ciento. En este caso, la tasa de desempleo aumenta a 5 por ciento.

crecerá 5\% durante el 2011, insertamos sus valores en la ecuación (2a) del apéndice metodológico A.2 y obtenemos una estimación para el PIB total del estado. Finalmente, al considerar este resultado podemos obtener una estimación de la tasa de desempleo al utilizar la ecuación (3a) del apéndice metodológico A.3. 


\section{Cuadro 1}

Efecto de las heladas sobre las variables económicas agregadas del estado de Sinaloa: predicciones del modelo econométrico

(Porcentaje)

\begin{tabular}{|l|c|c|}
\hline \multicolumn{1}{|c|}{ Variables } & $\begin{array}{c}\text { Simulación 1: sin } \\
\text { resiembra }\end{array}$ & $\begin{array}{c}\text { Simulación 2 y 3: } \\
\text { con resiembra }\end{array}$ \\
\hline Producción agrícola & -42.8 & -23.0 \\
\hline PIB primario & -28.0 & -15.1 \\
\hline PIB total & -6.4 & -2.5 \\
\hline Personas desempleadas & 5.7 & 5.0 \\
\hline
\end{tabular}

Fuente: elaboración propia.

\subsubsection{Predicciones Del MOdelo DE EQUILIBRIO GENERAL}

\subsubsection{Efectos agregados y por sectores económicos}

Con el fin de obtener efectos más desagregados, se especificó y calibró un modelo de equilibrio de corto plazo para la economía de Sinaloa que considera 30 sectores productivos, dos factores productivos (trabajo y capital), una familia representativa, dos niveles de gobierno (estatal y federal), el resto del país y un sector externo agregado. ${ }^{9}$ En el modelo el papel del gobierno federal es recabar impuestos (al consumo, al ingreso y a la producción) y dar transferencias al gobierno estatal, a sectores productivos específicos y a los hogares. Con los recursos que el gobierno federal le otorga, el gobierno del estado compra bienes y servicios diversos.

El efecto de las heladas sobre el PIB primario de Sinaloa que se estimó econométricamente, se introduce en el modelo de equilibrio general de corto plazo, de esta manera se determinan los efectos sobre: la producción interna por sector económico, el ingreso laboral y el ingreso del capital, el gasto en consumo y ahorro de los hogares, el nivel de ingreso y de gasto de los gobiernos federal y estatal, de manera simultánea; se toman en cuenta

\footnotetext{
${ }^{9}$ En el cuadro B1 del anexo metodológico B se encuentra el listado de los sectores incluidos en el modelo.
} 
las relaciones de dependencia entre la generación, distribución y asignación del ingreso (flujo circular) en Sinaloa. El mecanismo de transmisión de los principales efectos se expone en la figura $2 .^{10}$

Figura 2

Mecanismo de transmisión de efectos de las heladas a través de las variables del modelo de equilibrio general de corto plazo

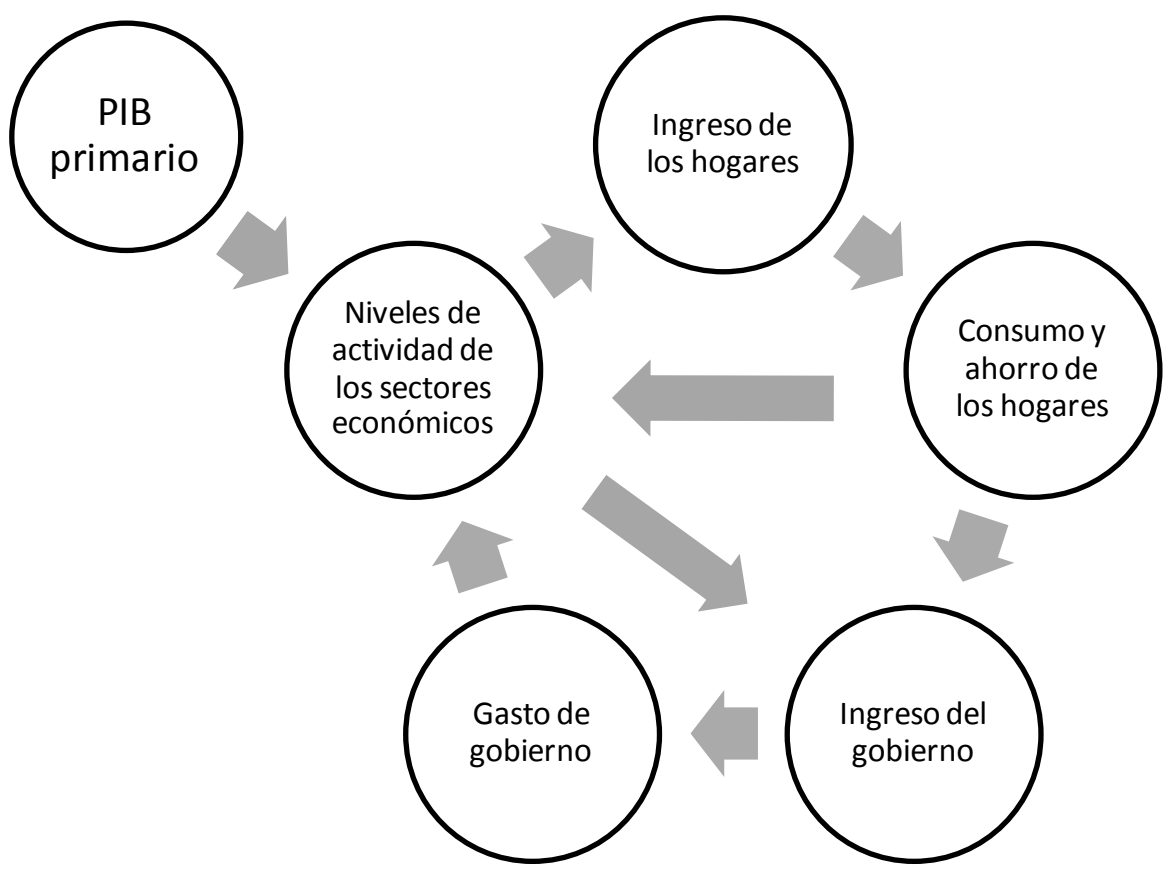

Fuente: elaboración propia.

En el caso de las simulaciones 1 y 2 se procede de la siguiente manera. El efecto de las heladas sobre la producción del sector agrícola se introduce como un choque exógeno en el modelo de equilibrio general, éste último se resuelve simultáneamente, y de esta forma se cuantifican los impactos finales sobre las variables antes mencionadas. En estas simulaciones se asume que el ingreso y el déficit del gobierno federal son

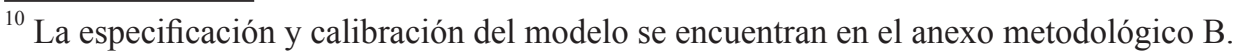


variables, pero mantiene el nivel de transferencias al gobierno estatal. Por lo tanto, el ingreso y gasto del gobierno estatal permanecen constantes. Nótese que en relación con la simulación 2 , el costo de la resiembra la financia el gobierno federal con más deuda o bien menos ahorro.

En la simulación 3 aplicamos las mismas reglas de cierre para los niveles de gobierno, pero, en este caso, el gobierno estatal compra grano para resembrar a un precio equivalente a $32 \%$ del precio al consumidor final, ${ }^{11}$ dichos recursos los obtiene al reducir su gasto en bienes y servicios.

\section{Simulación 1: Situación sin resiembra}

La producción agrícola disminuye $42.8 \%$, lo que impacta negativamente al PIB total en $6.6 \%$ (cuadro 2). Además de la agricultura, los sectores económicos que más se ven afectados en términos proporcionales son: industria textil; industria química; otras industrias manufactureras y productos metálicos, maquinaria y equipo. La baja en los niveles de actividad de dichos sectores se ubica entre $42.8 \%$ y $9.6 \%$ (cuadro 3 ). Mientras que las actividades productivas con las que se relacionan los efectos más grandes en magnitud absoluta son: servicios inmobiliarios; comercio; transporte y comunicaciones; industria alimentaria, de las bebidas y el tabaco y servicios de alojamiento temporal (Sus efectos suman $82 \%$ del impacto total sobre el PIB estatal. Ver cuadro 4)

$\mathrm{El}$ ingreso laboral se reduce $4.7 \% \mathrm{y}$ el ingreso del factor capital 8.2 por ciento. Esto implica una caída en el ingreso disponible de las familias sinaloenses, lo que provoca una disminución en el gasto en consumo y en el ahorro de las familias de 6.6 por ciento. En este sentido, el bienestar de la población del estado se reduce.

\section{Simulación 2: Situación con resiembra financiada por el gobierno federal}

El impacto se aminora con la política de resiembra, ya que el maíz es el principal cultivo de Sinaloa en términos de valor de producción. Bajo

\footnotetext{
${ }^{11}$ Promedio del porcentaje que representa el precio al productor de maíz de Sinaloa del precio al consumidor final durante el periodo 2004-2007, la diferencia es el margen de comercialización (SIAP, SAGARPA).
} 
tal escenario, la producción agrícola cae $23 \%$, lo cual se traduce en una reducción de $3.5 \%$ del PIB total del estado. Los sectores económicos con las mayores caídas en términos relativos y absolutos son los mismos que en la simulación 1.

En cuanto a ingreso, las remuneraciones o ingreso laboral y el ingreso del capital caen $2.5 \%$ y $4.4 \%$, respectivamente; de esta manera el gasto en consumo y el ahorro disminuyen $3.5 \%$, ya que las familias sinaloenses tienen menos ingreso.

Simulación 3: Situación con resiembra financiada por el gobierno estatal

Aquí la producción agrícola disminuye $23 \%$ y el gasto estatal en bienes y servicios $3.4 \%$, el PIB total baja 4.5 por ciento. En este sentido, el que el gobierno estatal financie la resiembra, en lugar del gobierno federal, genera una caída adicional de $1 \%$ en el PIB del estado.

Entre los sectores más afectados en términos relativos y absolutos se encuentran los principales proveedores de bienes y servicios para el gobierno: actividades del gobierno, servicios educativos y servicios médicos. En las simulaciones 1 y 2, 82\% del impacto en el PIB estatal provenía de la caída en el PIB de seis sectores económicos. Mientras que bajo la simulación 3, cuando se financia la resiembra con recursos estatales, $82 \%$ del impacto se explica por la baja en el PIB de ocho sectores económicos, entre ellos se encuentran los sectores proveedores del gobierno.

El ingreso laboral y el ingreso del sector privado caen $3.7 \%$ y $5.1 \%$, respectivamente. Nótese que en esta simulación, en relación con las otras dos, relativamente cae más el ingreso laboral. Esto es consecuencia de que los sectores económicos que le venden bienes y servicios al gobierno destinan una proporción alta de su valor agregado (sin impuestos) al pago al trabajo: actividades del gobierno destina $100 \%$, servicios de educación $80 \%$ y servicios médicos y de asistencia social 60 por ciento. Lo anterior impacta en el ingreso disponible y, con ello, en el gasto en consumo y el ahorro de las familias en 4.5 por ciento. 


\section{Cuadro 2}

Efecto de las heladas sobre las variables económicas agregadas del estado de Sinaloa: predicciones del modelo de equilibrio general

(Porcentaje)

\begin{tabular}{|l|c|c|c|}
\hline \multicolumn{1}{|c|}{ Variables } & $\begin{array}{c}\text { Simulación 1: } \\
\text { sin resiembra }\end{array}$ & $\begin{array}{c}\text { Simulación 2: resiembra } \\
\text { financiada por GF }\end{array}$ & $\begin{array}{c}\text { Simulación 3: resiembra } \\
\text { financiada por GE }\end{array}$ \\
\hline Consumo & $-6.6 \%$ & $-3.5 \%$ & $-4.5 \%$ \\
\hline Ahorro & $-6.6 \%$ & $-3.5 \%$ & $-4.5 \%$ \\
\hline PIB total & $-6.6 \%$ & $-3.5 \%$ & $-4.5 \%$ \\
\hline Ingreso del capital & $-8.2 \%$ & $-4.4 \%$ & $-5.1 \%$ \\
\hline Ingreso laboral & $-4.7 \%$ & $-2.5 \%$ & $-3.7 \%$ \\
\hline Producción doméstica & $-6.1 \%$ & $-3.3 \%$ & $-4.2 \%$ \\
\hline $\begin{array}{l}\text { Demanda intermedia } \\
\text { doméstica }\end{array}$ & $-5.3 \%$ & $-2.8 \%$ & $-3.7 \%$ \\
\hline $\begin{array}{l}\text { Demanda intermedia } \\
\text { de bienes del resto de } \\
\text { los estados }\end{array}$ & $-5.0 \%$ & $-2.7 \%$ & $-3.4 \%$ \\
\hline $\begin{array}{l}\text { Gasto de gobierno en } \\
\text { bienes y servicios }\end{array}$ & $0.0 \%$ & $0.0 \%$ & $-3.4 \%$ \\
\hline Recaudación & $-6.4 \%$ & $-3.4 \%$ & $-4.4 \%$ \\
\hline
\end{tabular}

Fuente: elaboración propia.

\section{Cuadro 3}

Efecto de las heladas en el PIB sectorial del estado de Sinaloa (Variaciones porcentuales)

\begin{tabular}{|l|c|c|c|}
\hline Variables & Simulación 1 & Simulación 2 & Simulación 3 \\
\hline Agricultura & $-42.8 \%$ & $-23.0 \%$ & $-23.0 \%$ \\
\hline Ganadería & $-3.5 \%$ & $-1.9 \%$ & $-2.4 \%$ \\
\hline $\begin{array}{l}\text { Aprovechamiento forestal, caza, } \\
\text { pesca y servicios relacionados }\end{array}$ & $-0.8 \%$ & $-0.4 \%$ & $-0.5 \%$ \\
\hline Minería & $-3.5 \%$ & $-1.9 \%$ & $-2.4 \%$ \\
\hline Electricidad, gas y agua & $-3.0 \%$ & $-1.6 \%$ & $-2.1 \%$ \\
\hline Construcción & $0.0 \%$ & $0.0 \%$ & $0.0 \%$ \\
\hline $\begin{array}{l}\text { Industria alimentaria, de las bebidas } \\
\text { y tabaco }\end{array}$ & $-5.0 \%$ & $-2.7 \%$ & $-3.4 \%$ \\
\hline
\end{tabular}




\begin{tabular}{|c|c|c|c|}
\hline Textiles & $-27.2 \%$ & $-14.6 \%$ & $-18.6 \%$ \\
\hline Industria de la madera & $-5.8 \%$ & $-3.1 \%$ & $-3.9 \%$ \\
\hline $\begin{array}{l}\text { Industria del papel y actividades } \\
\text { conexas }\end{array}$ & $-3.9 \%$ & $-2.1 \%$ & $-2.9 \%$ \\
\hline Química & $-17.7 \%$ & $-9.5 \%$ & $-12.1 \%$ \\
\hline $\begin{array}{l}\text { Fabricación de productos a base de } \\
\text { minerales no metálicos }\end{array}$ & $-6.9 \%$ & $-3.7 \%$ & $-4.7 \%$ \\
\hline Industrias metálicas básicas & $-4.4 \%$ & $-2.3 \%$ & $-3.0 \%$ \\
\hline $\begin{array}{l}\text { Productos metálicos, maquinaria y } \\
\text { equipo }\end{array}$ & $-9.6 \%$ & $-5.1 \%$ & $-6.5 \%$ \\
\hline $\begin{array}{l}\text { Fabricación de muebles y productos } \\
\text { relacionados }\end{array}$ & $-7.5 \%$ & $-4.0 \%$ & $-5.1 \%$ \\
\hline Otras industrias manufactureras & $-11.7 \%$ & $-6.3 \%$ & $-8.0 \%$ \\
\hline Comercio & $-3.4 \%$ & $-1.8 \%$ & $-2.3 \%$ \\
\hline Transporte y comunicaciones & $-5.0 \%$ & $-2.7 \%$ & $-3.4 \%$ \\
\hline Telecomunicaciones & $-6.9 \%$ & $-3.7 \%$ & $-4.8 \%$ \\
\hline Servicios financieros & $-5.8 \%$ & $-3.1 \%$ & $-4.8 \%$ \\
\hline Servicios inmobiliarios & $-6.1 \%$ & $-3.3 \%$ & $-4.2 \%$ \\
\hline $\begin{array}{l}\text { Servicios profesionales, científicos } \\
\text { y técnicos }\end{array}$ & $-5.6 \%$ & $-3.0 \%$ & $-4.2 \%$ \\
\hline $\begin{array}{l}\text { Dirección de corporativos y empre- } \\
\text { sas }\end{array}$ & $-4.2 \%$ & $-2.3 \%$ & $-2.9 \%$ \\
\hline Servicios de apoyo a los negocios & $-3.3 \%$ & $-1.8 \%$ & $-2.5 \%$ \\
\hline Servicios educativos & $-2.5 \%$ & $-1.3 \%$ & $-4.2 \%$ \\
\hline $\begin{array}{l}\text { Servicios médicos y de asistencia } \\
\text { social }\end{array}$ & $-3.1 \%$ & $-1.7 \%$ & $-4.0 \%$ \\
\hline Servicios artísticos & $-5.2 \%$ & $-2.8 \%$ & $-3.9 \%$ \\
\hline Servicios de alojamiento temporal & $-5.5 \%$ & $-3.0 \%$ & $-3.8 \%$ \\
\hline Otros servicios & $-4.3 \%$ & $-2.3 \%$ & $-3.0 \%$ \\
\hline Actividades del gobierno & $-0.1 \%$ & $-0.1 \%$ & $-4.1 \%$ \\
\hline
\end{tabular}

Fuente: elaboración propia. 


\section{Cuadro 4}

Distribución porcentual del efecto de las heladas en el PIB del estado de Sinaloa por sector económico

(Porcentaje)

\begin{tabular}{|c|c|c|c|}
\hline Variables & Simulación 1 & Simulación 2 & Simulación 3 \\
\hline Agricultura & $45.6 \%$ & $45.6 \%$ & $36.0 \%$ \\
\hline Ganadería & $0.6 \%$ & $0.6 \%$ & $0.6 \%$ \\
\hline $\begin{array}{l}\text { Aprovechamiento forestal, caza, pesca } \\
\text { y servicios relacionados }\end{array}$ & $0.5 \%$ & $0.5 \%$ & $0.4 \%$ \\
\hline Minería & $0.1 \%$ & $0.1 \%$ & $0.1 \%$ \\
\hline Electricidad, gas y agua & $0.9 \%$ & $0.9 \%$ & $0.9 \%$ \\
\hline Construcción & $0.0 \%$ & $0.0 \%$ & $0.1 \%$ \\
\hline $\begin{array}{l}\text { Industria alimentaria, de las bebidas y } \\
\text { tabaco }\end{array}$ & $5.1 \%$ & $5.1 \%$ & $5.1 \%$ \\
\hline Textiles & $0.4 \%$ & $0.4 \%$ & $0.5 \%$ \\
\hline Industria de la madera & $0.1 \%$ & $0.1 \%$ & $0.1 \%$ \\
\hline $\begin{array}{l}\text { Industria del papel y actividades } \\
\text { conexas }\end{array}$ & $0.2 \%$ & $0.2 \%$ & $0.2 \%$ \\
\hline Química & $0.9 \%$ & $0.9 \%$ & $0.9 \%$ \\
\hline $\begin{array}{l}\text { Fabricación de productos a base de } \\
\text { minerales no metálicos }\end{array}$ & $0.3 \%$ & $0.3 \%$ & $0.3 \%$ \\
\hline Industrias metálicas básicas & $0.2 \%$ & $0.2 \%$ & $0.2 \%$ \\
\hline $\begin{array}{l}\text { Productos metálicos, maquinaria y } \\
\text { equipo }\end{array}$ & $0.6 \%$ & $0.6 \%$ & $0.6 \%$ \\
\hline $\begin{array}{l}\text { Fabricación de muebles y productos } \\
\text { relacionados }\end{array}$ & $0.1 \%$ & $0.1 \%$ & $0.1 \%$ \\
\hline Otras industrias manufactureras & $0.1 \%$ & $0.1 \%$ & $0.1 \%$ \\
\hline Comercio & $10.4 \%$ & $10.4 \%$ & $10.4 \%$ \\
\hline Transporte y comunicaciones & $6.5 \%$ & $6.5 \%$ & $6.5 \%$ \\
\hline Telecomunicaciones & $2.0 \%$ & $2.0 \%$ & $2.0 \%$ \\
\hline Servicios financieros & $1.1 \%$ & $1.1 \%$ & $1.4 \%$ \\
\hline Servicios inmobiliarios & $11.4 \%$ & $11.4 \%$ & $11.5 \%$ \\
\hline $\begin{array}{l}\text { Servicios profesionales, científicos y } \\
\text { técnicos }\end{array}$ & $1.8 \%$ & $1.8 \%$ & $1.9 \%$ \\
\hline Dirección de corporativos y empresas & $0.0 \%$ & $0.0 \%$ & $0.0 \%$ \\
\hline
\end{tabular}




\begin{tabular}{|l|c|c|c|}
\hline Servicios de apoyo a los negocios & $0.6 \%$ & $0.6 \%$ & $0.6 \%$ \\
\hline Servicios educativos & $2.2 \%$ & $2.2 \%$ & $5.4 \%$ \\
\hline Servicios médicos y de asistencia social & $1.7 \%$ & $1.7 \%$ & $3.4 \%$ \\
\hline Servicios artísticos & $0.4 \%$ & $0.4 \%$ & $0.5 \%$ \\
\hline Servicios de alojamiento temporal & $3.2 \%$ & $3.2 \%$ & $3.2 \%$ \\
\hline Otros servicios & $2.9 \%$ & $2.9 \%$ & $2.9 \%$ \\
\hline Actividades del Gobierno & $0.1 \%$ & $0.1 \%$ & $4.1 \%$ \\
\hline Total & $100.0 \%$ & $100.0 \%$ & $100.0 \%$ \\
\hline
\end{tabular}

Fuente: elaboración propia.

\subsubsection{Multiplicadores de impacto}

Resulta interesante calcular lo que, en términos de la metodología insumo-producto, se denomina multiplicadores de impacto, que miden el efecto sobre una variable de interés, tal como la producción bruta, de una política o un choque exógeno, tomando en cuenta todos los efectos directos e indirectos generados por las relaciones intrínsecas entre los sectores económicos.

En nuestro caso, iremos un paso más allá, puesto que calcularemos los multiplicadores de impacto generados por las heladas en la economía del estado de Sinaloa, con base en un modelo de equilibrio general de corto plazo, que no sólo considera la relación entre las actividades productivas, sino que toma en cuenta las interrelaciones entre todos los agentes económicos.

En este sentido, calcularemos los multiplicadores de impacto sobre la producción bruta, valor agregado, ingreso laboral e ingreso del capital; bajo los tres escenarios analizados. Su cálculo es muy sencillo, por ejemplo, en el caso del multiplicador de impacto sobre la producción bruta (MIQ):

$$
M I Q=\frac{\left.Q\right|_{\text {Equilibrio escenario } 1}-\left.Q\right|_{\text {Equilibrio inicial }}}{\text { Cambio en la producción agrícola del escenario } 1}
$$


Notemos que el multiplicador lo que calcula es el cambio en la producción agregada de la economía ante el cambio en la variable de política o, en este caso, la variable que está siendo impactada por el choque.

En el mismo sentido, el multiplicador de impacto sobre el valor agregado (MVA), el ingreso laboral (MIL) y el ingreso del capital (MIK) se calculan aplicando la misma fórmula, sólo cambiamos la producción bruta por la variable correspondiente.

Cabe comentar que bajo las simulaciones 1 y 2 se obtendrán los mismos multiplicadores de impacto, ya que sólo difieren en el monto del choque sobre la producción agrícola. Mientras que la simulación 3 esperamos arroje multiplicadores más altos, dado que el gasto en bienes y servicios del gobierno estatal varía. En este sentido, la diferencia entre los multiplicadores de impacto de la simulación 3 , en relación con los multiplicadores de las simulaciones 1 y 2 , se puede interpretar como la parte de los multiplicadores de impacto que se explica por el cambio en el gasto en bienes y servicios del gobierno estatal.

Los resultados indican que el choque de las heladas sobre la producción agrícola tiene un impacto relevante en la economía de Sinaloa. Bajo los escenarios 1 y 2 , a cada peso de caída en la producción agrícola del estado de Sinaloa se asocian reducciones de: 2.51 pesos de producción bruta, 1.68 pesos de valor agregado, 55 centavos de ingreso laboral y 1.18 pesos de ingreso de capital. Ver cuadro 5.

Como se esperaba, los multiplicadores de impacto del choque de las heladas sobre la producción agrícola, combinado con una política de resiembra financiada por el gobierno estatal mediante una política de redirección del gasto en bienes y servicios, son más altos. Bajo el escenario 3, a cada peso de caída en la producción agrícola de Sinaloa se asocian reducciones de: 3.18 pesos de producción bruta, 2.13 pesos de valor agregado, 83 centavos de ingreso laboral y 1.37 pesos de ingreso del capital.

Nótese que, en términos proporcionales, el multiplicador de impacto sobre el ingreso laboral es el que más se incrementa cuando el gasto en bienes y servicios del gobierno estatal varía. De 55 centavos pasa a 83 centavos, lo cual representa un aumento de $49.5 \%$ en el multiplicador. Ello se debe, como lo habíamos comentado anteriormente, a que los sectores que proveen bienes y servicios para el gobierno utilizan intensivamente mano de obra. 


\section{Cuadro 5}

Multiplicadores de impacto sobre producción bruta, valor agregado, ingreso laboral e ingreso de capital

\begin{tabular}{|l|c|c|c|c|c|}
\hline Multiplicadores & Simulación 1 & Simulación 2 & Simulación 3 & $\begin{array}{c}\text { Efecto del } \\
\text { gobierno } \\
\text { (absoluto) }\end{array}$ & $\begin{array}{c}\text { Efecto del } \\
\text { gobierno } \\
\text { (porcentual) }\end{array}$ \\
\hline MQ & 2.51 & 2.51 & 3.18 & 0.68 & $26.9 \%$ \\
\hline MVA & 1.68 & 1.68 & 2.13 & 0.45 & $26.8 \%$ \\
\hline MIL & 0.55 & 0.55 & 0.83 & 0.27 & $49.5 \%$ \\
\hline MIK & 1.18 & 1.18 & 1.37 & 0.19 & $16.1 \%$ \\
\hline
\end{tabular}

Fuente: elaboración propia.

\section{CONCLUSIONES}

Los principales resultados de las estimaciones econométricas indican que la pérdida de la producción total de maíz genera una caída de $42.8 \%$ en la producción agrícola del estado, lo cual se traduce en una baja de $6.4 \%$ del PIB estatal y un aumento en la tasa de desempleo de 5.7 por ciento. Mientras que la resiembra de $60 \%$ de las hectáreas de maíz siniestradas permite disminuir el impacto negativo sobre la producción agrícola a $23.0 \%$; sobre el producto interno bruto a $2.5 \%$ y sobre la tasa de desempleo a 5.0 por ciento.

Al introducir la baja de $42.8 \%$ de la producción agrícola en el modelo de equilibrio general de corto plazo se obtiene una caída en el PIB estatal ligeramente mayor al modelo econométrico, $6.6 \%$, la cual se trasfiere completamente al ingreso, consumo y ahorro de las familias de Sinaloa. Bajo la política de resiembra financiada con recursos federales, el impacto sobre el PIB y el ingreso, consumo y ahorro de los hogares se reduce a $3.5 \%$ (un punto porcentual mayor al modelo econométrico).

Los resultados indican que el choque de las heladas sobre la producción agrícola tiene un impacto relevante en la economía del estado de Sinaloa. A cada peso de caída en la producción agrícola de dicho estado se asocian reducciones de: 2.51 pesos de producción bruta, 1.68 pesos de valor agregado, 55 centavos de ingreso laboral y 1.18 pesos de ingreso de capital. 
Si la resiembra es financiada con una política de redirección del gasto estatal, el PIB de Sinaloa se reduce $4.5 \%, 1 \%$ más que con resiembra financiada con recursos federales. Lo anterior es consecuencia de que la resiembra financiada con recursos estatales implica una reducción de 3.4\% en el gasto en servicios públicos, como son servicios educativos, médicos $\mathrm{y}$ actividades del gobierno.

En este sentido, en la medida que se tome en cuenta el efecto económico del financiamiento de una política, los efectos netos de la política se reducen. Si se asume una política de resiembra financiada por el gobierno estatal mediante una política de redirección del gasto para contrarrestar los efectos de las heladas en la producción agrícola, se obtiene que a cada peso de caída en la producción agrícola se asocian reducciones de: 3.18 pesos de producción bruta, 2.13 pesos de valor agregado, 83 centavos de ingreso laboral y 1.37 pesos de ingreso del capital.

Cabe comentar que los efectos arrojados por el modelo de equilibrio general son de corto plazo, antes de que cambien los precios de los productos y de los factores productivos ante el choque y, en consecuencia, se desate la redistribución de factores productivos entre sectores económicos. Lo cual generaría que el monto de los efectos cambie. Además, estamos manteniendo la inversión en el estado fija, aunque el ahorro familiar, del gobierno federal y del sector externo están variando. Por ello, deben tomarse como una primera aproximación a los efectos del choque. 


\section{BIBLIOGRAFÍA}

Armington, P. 1969. "A Theory of Demand for Products Distinguished by Place of Production", International Monetary Fund Staff Papers, marzo, pp. 159-176.

Balassa, B. 1965. "Trade liberalization and revealed comparative advantage", The Manchester School of Economics and Social Science, 33: 99-123.

Boyd, R. y M.E. Ibarrarán. 2011. "El costo del cambio climático en México: análisis de equilibrio general de la vulnerabilidad intersectorial”, Gaceta de Economía, año 16, número especial, tomo I: 115-133.

Diersen, M.A., G. Taylor y A. May. 2002. "Direct and Indirect Effects of Drought on South Dakota's Economy", Economics Commentator, núm. 432, agosto.

Diersen, M.A. y G. Taylor. 2003. "Examining Economic Impact and Recovery in South Dakota from the 2002 Drought", Department of Economics, South Dakota State University, Economics Staff Paper, núm. 8.

Flegg A. T. y C.D.Webber. 1997. "On the appropiate use of location quotients in generating regional input-output tables: reply", Regional Studies, 31, pp. 795-805.

Hodges, A.W. y J.J. Haydu. 2003. "Economic Impacts of Drought on the Florida Environmental Horticulture Industry", Department of Food and Resource Economics, University of Florida, EDIS document FE385, <http://edis.ifas.ufl.edu/pdffiles/FE/FE38500. pdf $>$

Horridge, M., J. Madden y G. Wittwer. 2005. "The Impact of the 20022003 drought on Australia", Journal of Policy Modelling, 27(3): 285-303.

INEGI. Varios años. Encuesta nacional de empleo urbano, México.

— Varios años. Encuesta nacional de ocupación y empleo, México. 2003. Finanzas públicas estatales y municipales, México. 
2006. Anuario estadístico por entidad federativa, México.

2008. Matriz insumo producto 2003, sistema de cuentas nacionales de México, México.

_ Varios años. Producto interno bruto nacional y estatal, series anuales, Banco de información económica, México.

Leontief, W. 1965. "The Structure of the U.S. Economy”, Scientific American, abril, pp. 25-35.

Okun, A. 1962. "A Potential GNP: its measurement and significance", Proceedings of the Business and Economic Statistics Section of the American Statistics Association, pp. 98-104.

Pyatt, G. y J.I. Round. 1979. "Accounting and Fixed Price Multipliers in a Social Accounting Matrix Framework", The Economic Journal, 89(356): 850-873.

SAGARPA. Varios años. Márgenes de comercialización del maíz en Sinaloa, México, SIAP.

Varios años. Valor de la producción de los principales cultivos de Sinaloa, México, SIAP.

Shoven, J. y J. Walley. 1973. “A General Equilibrium with Taxes: A Computational Procedure and an Existence Proof", Review of Economics Studies, 40(124): 475-495.

Shoven, J. y J. Walley. 1972. "A General Calculation of the Effects of Diferential Taxation of Income from Capital in the US", Journal of Public Economics, 1(3/4): 281-321.

Stone, R. 1985. "The Disaggregation of the Household Sector in the National Accounts", en G. Pyatt y J.I. Round (comps.), Social Accounting Matrices: a Basis for Planning, The World Bank, Washington.

Wheaton, E., S. Kulshreshtha, V. Wittrock y G. Koshida. 2008. "Dry times: hard lessons from the Canadian drought of 2001 and 2002", The Canadian Geographer/Le Géographe canadien, 52(2): 241-262. 


\section{ANEXO METODOLÓGICO}

\section{A. MODELOS ECONOMÉTRICOS PARA LA ECONOMÍA DE SINALOA}

\section{A.1 Modelo econométrico del PIB primario de Sinaloa}

Para poder predecir el efecto de la producción agrícola sobre el PIB primario de la entidad corrimos una regresión doble logarítmica entre estas dos variables. La ecuación es:

$$
\log \left(P I B P R_{t}\right)=\alpha_{0}+\alpha_{1} \log \left(V P A R_{t}\right)+u_{t}
$$

donde PIBPR es el PIB primario a pesos del año 2003 de Sinaloa, VPAR es el valor de la producción agrícola a precios del 2003 de los principales diez cultivos de Sinaloa, " $t$ " representa el año, se considera de 1993 al 2010, $u$ es el residual y los coeficientes $\alpha$ son los parámetros del modelo. Las pruebas de bondad de ajuste muestran que la producción de estos cultivos explica casi $95 \%$ de la variabilidad del PIB primario del estado. ${ }^{12}$ El coeficiente que mide el efecto del valor de la producción sobre el PIB primario de Sinaloa es significativo ( $\mathrm{p}<0.01$ ), e indica que un cambio de $1 \%$ en el valor de la producción de los principales cultivos se transfiere en $0.6544 \%$ de crecimiento al PIB primario (Ver cuadro A1).

\footnotetext{
${ }^{12}$ La prueba Engle-Granger sobre los residuales de la regresión sugiere que éstos son estacionarios $(\mathrm{t}=-5.97) \mathrm{y}$, por lo tanto, la relación entre el valor de la producción de los principales cultivos y el PIB primario no es espuria. También, la evidencia es consistente con la ausencia de correlación serial de los residuales $(\mathrm{DW}=1.7)$.
} 


\section{Cuadro A1}

Regresión del logaritmo del PIB primario a pesos constantes de 2003

\begin{tabular}{lcccc}
\hline & Coeficiente & Error Estándar $\mathrm{t}$ & P-value \\
\hline Constante & 1.646094 & 0.966723 & 1.70276 & 0.1092 \\
Log(VPAR) & 0.654425 & 0.042045 & 15.5647 & 0 \\
& & & & \\
R2 & 0.941693 & $\mathrm{~F}$ & 242.261 & \\
R2 ajustado & 0.937806 & $\mathrm{P}(\mathrm{F})$ & 0 & \\
Suma de Errores & & & & \\
al Cuadrado & 0.02373 & & & \\
\hline
\end{tabular}

Fuente: elaboración propia.

\section{A.2. Modelo econométrico del PIB total de Sinaloa}

Se decidió modelar el efecto del PIB primario sobre la economía del estado, corrimos la regresión doble logarítmica entre el PIB total del estado contra el PIB primario y el PIB de todo el país. Es decir, nuestro modelo asume que el estado de Sinaloa está determinado por la dinámica agrícola, sector donde se demuestra que el estado tiene clara ventaja competitiva, y la dinámica del país. La ecuación que describe el modelo es:

$$
\log \left(P I B S_{t}\right)=\beta_{0}+\beta_{1} \log \left(P I B P R_{t}\right)+\beta_{1} \log \left(P I B N_{t}\right)+v_{t}
$$

donde PIBS es el PIB total a pesos de 2003 del estado de Sinaloa, el PIBPR es el PIB primario a pesos de 2003 de Sinaloa, PIBN es el PIB total nacional a pesos de 2003, " $t$ " representa el año, se considera de 1993 a 2010, $v$ es el residual y los coeficientes $\beta$ son los parámetros del modelo. El cuadro A2 muestra los resultados de esta regresión. 
Cuadro A2

Regresión del logaritmo del PIB total de Sinaloa a pesos constantes de 2003

\begin{tabular}{lccrc}
\hline & Coeficiente & Error Estándar $\mathrm{t}$ & P-value \\
\hline Constante & 4.190987 & 0.813592 & 5.15122 & 0.0001 \\
Log(PIBPR) & 0.303279 & 0.071686 & 4.23064 & 0.0007 \\
Log(PIBN) & 0.421151 & 0.080648 & 5.22211 & 0.0001 \\
& & & & \\
R2 & 0.979954 & $\mathrm{~F}$ & 366.643 & \\
R2 ajustado & 0.977281 & $\mathrm{P}(\mathrm{F})$ & \multicolumn{2}{c}{0} \\
Suma de Errores & & & & \\
al Cuadrado & 0.004926 & & & \\
\hline
\end{tabular}

Fuente: elaboración propia.

Nuevamente, el modelo propuesto sugiere que las dinámicas del sector primario y de la economía del país explican $97 \%$ de las variaciones del PIB total del estado de Sinaloa. También los residuales parecen ser estacionarios ( $\mathrm{t}=-3.86)$, lo que indica que la relación entre estas variables es estable y no espuria. No hay evidencia que indique autocorrelación serial de los residuales, lo que nos lleva a descartar especificaciones dinámicas más complicadas que la presentada aquí.

Ambos coeficientes son significativamente distintos de cero $(\mathrm{p}<0.01)$, positivos, como esperaríamos, es decir, al expandirse el sector primario y/o la economía el PIB total de Sinaloa crece. La magnitud del coeficiente del PIB primario es interesante, ronda el 0.3 , que es poco más del doble de la participación de este sector en la economía. Es decir, tal y como se comentó antes al crecer el sector primario la economía de Sinaloa crece por dos vías: la primera por el efecto directo de la agricultura en el PIB, la segunda por los efectos multiplicadores en la producción de los otros sectores animados por la expansión de ingresos que ocasiona la expansión agrícola. Esto es, si se uutilizara sólo la participación del sector en la economía para hacer el pronóstico del efecto de este sector en la economía total estaríamos claramente subestimando el efecto. 


\section{A.3 Modelo econométrico de la tasa de desempleo de Sinaloa}

La serie de la tasa de desempleo de 1993 a 2004 corresponde a la estimación de la proporción de la población económicamente activa (PEA) que se encontraba desempleada en Culiacán de acuerdo con la encuesta nacional de empleo urbano de INEGI. Del año 2005 al año 2010 corresponde a la tasa de desempleo para todo el estado, estimada a través de la encuesta nacional de ocupación y empleo.

Decidimos probar una especie de Ley de Okun modificada para el caso de Sinaloa, para ello se corrió la regresión del cambio en la tasa de desempleo en la entidad contra la tasa de crecimiento del PIB estatal. Una variante del modelo de regresión consistió en incluir una variable dicotómica que toma el valor 1 para los años de 2005 a 2010 y 0 entre 1993 y 2004, misma que pretende capturar efectos de desplazamiento a raíz del cambio en la metodología del cálculo del desempleo, pero como no resultó significativa no se incluyeron en la versión final. La ecuación (3a) describe la ecuación para el desempleo:

$$
\Delta\left(T D S_{t}\right)=\delta_{0}+\delta_{1} \Delta \log \left(P I B S_{t}\right)+e_{t}
$$

donde $\Delta$ es el operador de primera diferencia, TDS es la tasa de desempleo abierto en Sinaloa y $\Delta \log (\mathrm{PIBS})$ puede interpretarse como la tasa de crecimiento del PIB a precios constantes del estado de Sinaloa, “t”" es el año (1993 a 2010) y $e$ es el residual de la regresión. Los resultados se presentan en el cuadro A3. ${ }^{13}$

\footnotetext{
${ }^{13}$ Conviene apuntar que se hicieron pruebas para estimar las ecuaciones (1), (2) y (3) como un sistema al asumir que los residuales ( $\mathrm{u}, \mathrm{v}$ y e) de las tres ecuaciones estuvieran correlacionados, se estima el sistema por el método SUR, por sus siglas en inglés (System of Seemingly Unrelated Regression Equations). Como los coeficientes no cambian en absoluto, decidimos usar los coeficientes reportados en los cuadros A1, A2 y A3 para realizar las simulaciones.
} 


\section{Cuadro A3}

Regresión del cambio de la tasa de desempleo en Sinaloa

\begin{tabular}{lcrrrr}
\hline & Coeficiente & \multicolumn{2}{c}{ Error Estándar t } & \multicolumn{1}{c}{ P-value } \\
\hline Constante & 0.004351 & & 0.002327 & 1.86964 & 0.0812 \\
$\Delta \log ($ PIBS) & -0.171509 & & 0.065411 & -2.62202 & 0.0192 \\
& & & & & \\
R2 & 0.314285 & $\mathrm{~F}$ & & 6.87498 & \\
R2 ajustado & 0.268571 & $\mathrm{P}(\mathrm{F})$ & & 0.01924 & \\
Suma de Errores & & & & & \\
al Cuadrado & 0.001026 & & & & \\
\hline
\end{tabular}

Fuente: elaboración propia.

La variable dependiente es el cambio en la tasa desempleo abierto, la independiente es la tasa de crecimiento del PIB total de Sinaloa. La R2 de la regresión es menor a las de las otras regresiones, ya que las variables en cuestión son estacionarias, es decir, no presentan tendencia alguna, lo que hace más difícil explicar su volatilidad. Pero aun así, el ajuste es aceptable $(\mathrm{F}=6.87, \mathrm{p}<0.05)$. Los residuales también son estacionarios $(\mathrm{t}=-6.21)$, lo que indica que la relación es estable. Asimismo, no hay evidencia de autocorrelación serial, así que no es recomendable incluir rezagos como regresores.

El coeficiente es significativamente diferente de cero $(p<0.05)$ e indica una relación inversa entre ambas variables, lo cual es consistente con la evidencia internacional. La relación indica que si aumenta la tasa de crecimiento en la economía de Sinaloa, la tasa de desempleo disminuye, más concretamente, por cada $1 \%$ adicional de crecimiento en el estado, la tasa de desempleo se reduce en casi $1 / 5$ parte o 0.17 por ciento. 


\section{B. MODELOS MULTISECTORIALES PARA LA ECONOMÍA DE SINALOA}

\section{B.1 Modelo de equilibrio general aplicado de corto plazo}

\section{B.1.1 Especificación del modelo}

El modelo considera 30 sectores productivos, un tipo de familia y dos factores productivos (trabajo y capital), contenidos en el cuadro B1.

La producción total $\left(Q_{j}\right)$ es una función anidada en tres niveles. En el primer nivel se combina producción doméstica $\left(\mathrm{Y}_{\mathrm{j}}\right)$ e importaciones $\left(\mathrm{M}_{\mathrm{j}}\right)$ para obtener la producción total mediante una función Cobb Douglas con rendimientos constantes a escala, esta función es también denominada Agregador de Armington (Armigton, 1969). En el segundo nivel se determina la producción doméstica, se utilizan bienes intermedios $\left(\mathrm{x}_{\mathrm{ij}}\right)$ y valor agregado $\left(\mathrm{VA}_{\mathrm{j}}\right.$ ) en proporciones fijas mediante una función tipo Leontief. Finalmente, para permitir sustitución entre factores productivos, trabajo y capital $\left(\mathrm{L}_{\mathrm{j}} \mathrm{y} \mathrm{K}_{\mathrm{j}}\right)$ el valor agregado se modela como una función Cobb Douglas con rendimientos constantes a escala:

$$
\begin{gathered}
Q_{j}=\beta_{j} Y_{j}^{b_{j}} M_{j}^{1-b_{j}} \\
Y_{j}=\operatorname{Min}\left\{\frac{x_{i j}}{a_{i j}}, \ldots, \frac{x_{9_{j}}}{a_{9_{j}}}, \frac{V A_{j}}{v_{j}}\right\} \\
V A_{j}=A_{j} K_{j}^{\alpha_{j}} L_{j}^{1-\alpha_{j}} \\
\text { para j,i }=1,2,3, \ldots, 30 .
\end{gathered}
$$

Al suponer que los productores minimizan costos, las demandas de producción doméstica, importaciones, bienes intermedios, valor agregado $\mathrm{y}$ factores productivos son:

$$
Y j=\frac{Q_{j}}{\beta_{j}}\left[\frac{b_{j}}{1-b_{j}} \frac{P_{m_{j}}}{P_{j}}\right]^{1-b_{j}}
$$




$$
\begin{gathered}
M_{j}=\frac{Q_{j}}{\beta_{j}}\left[\frac{b_{j}}{1-b_{j}} \frac{P_{m_{j}}}{P_{j}}\right]^{-b_{j}} \\
x_{i j}=a_{i j} * Y_{j} \\
V A_{j}=v_{j}^{*} Y_{j} \\
L_{j}=\frac{V A_{j}}{A_{j}}\left[\frac{\alpha_{j}}{1-\alpha_{j}} \frac{w}{r}\right]^{-\alpha_{j}} \\
K_{j}=\frac{V A_{j}}{A_{j}}\left[\frac{\alpha_{j}}{1-\alpha_{j}} \frac{w}{r}\right]^{\left(1-\alpha_{j}\right)} \\
\text { para } \mathrm{j}=1,2,3, \ldots, 30 .
\end{gathered}
$$

donde $\mathrm{P}_{\mathrm{mj}}$ es el precio de los bienes importados del sector $j, \mathrm{P}_{\mathrm{j}}$ es el precio interno de los bienes del sector $j$, w es el salario y r el rendimiento del capital. Se supone que el salario y la renta de capital son iguales para todos los sectores.

El ingreso de las familias (RENTA) proviene de la venta de sus dotaciones de trabajo y capital:

$$
R E N T A=w L+r K
$$

Las preferencias del hogar representativo son descritas por una función de utilidad Cobb Douglas que depende del consumo (C) y ahorro (Ah). Se supone que el hogar representativo sigue un proceso de optimización para elegir cuánto consumir hoy y cuánto ahorrar sujeto a su ingreso disponible:

$\operatorname{Max} \mathrm{U}=\mathrm{C}^{\mathrm{g}} \mathrm{Ah}^{1-\gamma}$

s. a. IPC * C +IPI*Ah=RENTA* $(1-t r)$,

donde $\operatorname{tr}=$ impuesto sobre la renta, IPC $=$ nivel general de precios al consumidor, tc=impuesto sobre el valor agregado, e IPI es el índice de precios al inversionista.

Del anterior proceso de optimización se obtienen las ecuaciones de demanda: 


$$
\begin{gathered}
C=\frac{\alpha * i n g(1-t r)}{I P C^{*}(1+t c)} \\
A h=\frac{\beta * i n g(1-t r)}{I P I}
\end{gathered}
$$

Con respecto al gobierno su supone lo siguiente. El gobierno federal recauda impuestos al consumo, al ingreso y a la producción y da transferencias al gobierno estatal. Mientras que el gobierno estatal utiliza dichas transferencias para comprar bienes y servicios públicos que son otorgados a los hogares. Desafortunadamente no se contó con suficiente información para desagregar los impuestos locales.

Puesto que se trata de un modelo de cantidades, los precios relativos se mantienen fijos. Por lo tanto, es plausible suponer que el resto de la demanda final $\left(\mathrm{RDF}_{\mathrm{j}}\right)$ se mantiene constante. Entonces, la demanda total $\left(\mathrm{D}_{\mathrm{i}}\right)$ para cada sector $i$ viene dada por:

$$
\begin{gathered}
D_{i}=D I_{i}+C_{i}+G_{i}+R D F_{i} \\
D I_{i}=\sum_{j=1}^{30} x_{i j} \\
G_{i}=\eta_{i} G T \\
\text { para } \mathrm{i}=1,2,3, \ldots, 30 .
\end{gathered}
$$

donde $\varphi_{i}$ denota la proporción de la cantidad del bien de consumo que se produce con bienes vendidos por el sector $i$, por lo tanto, $\mathrm{C}_{\mathrm{i}}$ son las ventas totales de bienes para consumo privado del sector $i$. En el mismo sentido, $\eta_{i}$ es la proporción del gasto total del gobierno estatal que se destina a la compra de bienes y servicios provistos por el sector $i$.

En el equilibrio, para cada sector, la producción total debe ser igual a la demanda final. Este supuesto introduce la simultaneidad entre las elecciones de producción y de consumo en la economía:

$$
Q_{i}=D_{i}
$$

para $i=1,2,3, \ldots, 30$. 


\section{Cuadro B1}

Lista de sectores económicos

\begin{tabular}{|c|l|c|l|}
\hline AE1 & Agricultura & AE16 & Otras industrias manufactureras \\
\hline AE2 & Ganadería & AE17 & Comercio \\
\hline AE3 & $\begin{array}{l}\text { Aprovechamiento forestal, caza, } \\
\text { pesca y servicios relacionados }\end{array}$ & AE18 & Transporte y comunicaciones \\
\hline AE5 & Electricidad, gas y agua & AE19 & Telecomunicaciones \\
\hline AE6 & Construcción & AE20 & Servicios financieros \\
\hline AE7 & $\begin{array}{l}\text { Industria alimentaria, de las bebidas } \\
\text { y tabaco }\end{array}$ & AE21 & Servicios inmobiliarios \\
\hline AE8 & Textiles & $\begin{array}{l}\text { Servicios profesionales, científicos y } \\
\text { técos }\end{array}$ \\
\hline AE9 & Industria de la madera & AE23 & Dirección de corporativos y empresas \\
\hline AE10 & $\begin{array}{l}\text { Industria del papel y actividades } \\
\text { conexas }\end{array}$ & AE24 & Servicios de apoyo a los negocios \\
\hline AE11 & Química & Servicios educativos \\
\hline AE12 & $\begin{array}{l}\text { Fabricación de productos a base de } \\
\text { minerales no metálicos }\end{array}$ & AE27 & Servicios artísticos \\
\hline AE13 & Industrias metálicas básicas & AE28 & Servicios de alojamiento temporal \\
\hline AE14 & $\begin{array}{l}\text { Productos metálicos, maquinaria y } \\
\text { equipo }\end{array}$ & AE29 & Otros servicios \\
\hline AE15 & $\begin{array}{l}\text { Fabricación de muebles y productos } \\
\text { relacionados }\end{array}$ & AE30 & Actividades del gobierno \\
\hline
\end{tabular}

Fuente: elaboración propia.

\section{B.1.2 Calibración}

El modelo de equilibrio general de corto plazo fue calibrado con base en una matriz de contabilidad social para el estado de Sinaloa, que describe las relaciones de ingreso y gasto entre: un tipo de hogar representativo, dos niveles de gobierno (estatal y federal), 30 sectores económicos o actividades productivas, un tipo de trabajo, un tipo de capital y un sector externo agregado. Para construirla se derivó una matriz insumo producto del estado de Sinaloa para el año 2003 (MIPS 2003), su construcción 
se describe en el siguiente apartado. Los datos de finanzas públicas del gobierno del estado de Sinaloa provienen de la base de datos del INEGI que se denomina finanzas públicas estatales y municipales, en tanto que la recaudación de impuestos federales en el estado de Sinaloa proviene de la publicación del anuario estadístico por entidad federativa, edición 2006, del INEGI. El esquema de la matriz se encuentra en el cuadro B2 (por razones de espacio no se presenta la matriz de contabilidad social, pero puede ser solicitada a los autores). 


\begin{tabular}{|c|c|c|c|c|c|c|c|c|c|c|}
\hline \multirow{9}{*}{ 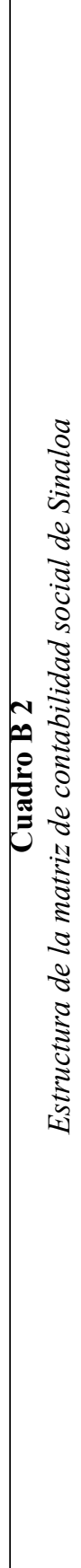 } & 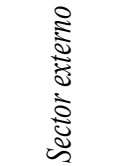 & & & & 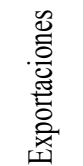 & & & & & \\
\hline & 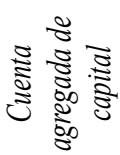 & & & & & 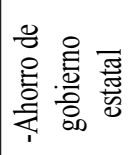 & 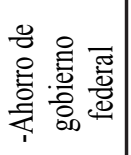 & & 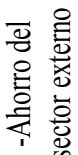 & \\
\hline & 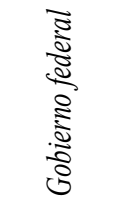 & 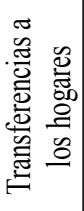 & & & & 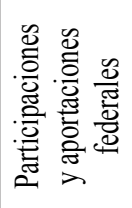 & & & & \\
\hline & 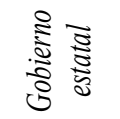 & & & & $\begin{array}{ll}0 & 0 \\
0 & 0 \\
0 & 0 \\
0 & 0 \\
0 & 0\end{array}$ & & & & & \\
\hline & 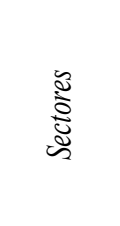 & & 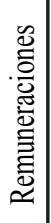 & 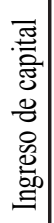 & 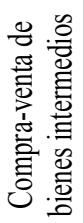 & & 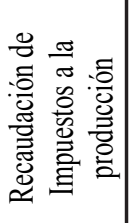 & & 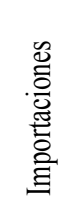 & \\
\hline & 胥 & 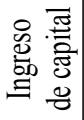 & & & & & & & & \\
\hline & 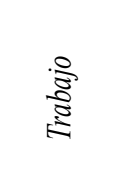 & 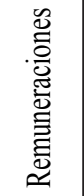 & & & & & & & & \\
\hline & 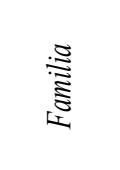 & & & & & 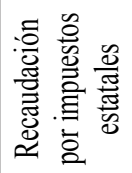 & 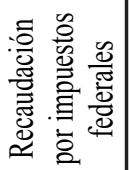 & 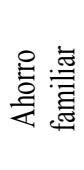 & & $\begin{array}{l}\frac{\pi}{2} \\
\frac{2}{0} \\
\frac{0}{2} \\
: 0\end{array}$ \\
\hline & 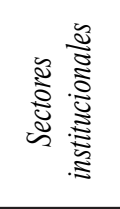 & 䄈 & 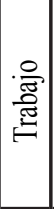 & 荵 & 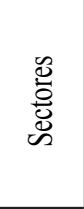 & 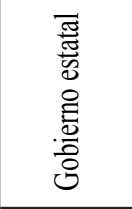 & 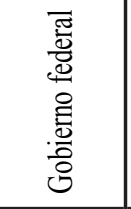 & 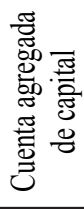 & 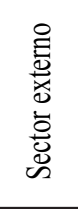 & $\begin{array}{l}\frac{\pi}{0} \\
\frac{0}{\pi} \\
0 \\
\ddot{0} \\
\stackrel{0}{0} \\
\stackrel{0}{0}\end{array}$ \\
\hline
\end{tabular}

Revista de Economía - Vol. XXX - Núm. 81 
B.1.3 Construcción de la matriz insumo producto del estado de Sinaloa (MIPS 2003)

La matriz insumo producto es una tabla de doble entrada que engloba las relaciones de compra-venta intersectoriales. Describe cómo se genera la producción bruta sectorial, tanto por el lado de la utilización (demanda) como de los recursos (oferta). En este sentido es una herramienta muy útil para establecer modelos de producción para una economía específica.

Construir matrices insumo producto (MIP) en nivel regional es muy costoso en tiempo y recursos, por ello, generalmente se aplican métodos indirectos de regionalización para derivar estas bases de datos. Los métodos de regionalización consisten en calcular la MIP regional a partir de la MIP nacional, al aplicar ciertos supuestos y utilizar la información disponible que sea representativa para la región en cuestión.

Por tal motivo, para construir la matriz insumo producto del estado de Sinaloa (MIPS) aplicamos el método de Flegg (FLQ) a la MIP nacional 2003, publicada por el INEGI, agregada a 30 sectores económicos. Este método de regionalización tiene la virtud de permitirnos obtener coeficientes técnicos específicos para Sinaloa, es decir, podemos identificar las compras $\mathrm{y}$ ventas de bienes intermedios producidos y consumidos en dicho estado (conocida como matriz de transacciones intersectoriales). A continuación se describe cómo derivamos cada una de las partes de la MIPS 2003.

\section{B.1.3.1 Matriz de transacciones intersectoriales: método de Flegg}

Este método asume que las técnicas de producción nacionales y regionales son iguales $\mathrm{y}$, por ello, los coeficientes técnicos nacionales $\left(a_{i j}{ }^{N}\right)$ de la MIP en nivel país y en nivel región también lo son. Por lo tanto, el consumo intermedio de origen nacional del sector $j$ en Sinaloa $\left(C I T^{R}\right)$ es igual:

$$
C I T_{j}^{R}=\sum_{i=1}^{24} a_{i j}{ }^{N} * P B_{j}^{R}
$$

$\mathrm{PB}_{\mathrm{j}}{ }^{\mathrm{R}}=$ Producción bruta a precios del productor del sector $j$ en el estado de Sinaloa. 
En la medida que se desee derivar una MIP con coeficientes técnicos regionales $\left(a_{i j}{ }^{R}\right)$, entonces los $a_{i j}{ }^{N}$ son ajustados mediante los coeficientes de localización (conocidos por sus siglas en inglés $L Q$ ), que identifican si los bienes intermedios del sector $i$ necesarios para producir en el sector $j$ son provistos por la misma región o son importados del resto de los estados. Los coeficientes de localización $\left(F L Q_{i j}\right)$ que utilizaremos son los desarrollados por Flegg y Webber (1997) y se calculan de la siguiente manera:

$$
\begin{gathered}
F L Q_{i j}=C I L Q_{i j}{ }^{*} \lambda \\
\lambda=\left[\log _{2}\left(1+\frac{P I B^{R}}{P I B^{N}}\right)\right]^{\delta} \\
S L Q_{i}=\frac{P I B_{i}^{R} / P I B^{R}}{P I B_{i}^{N}} / P I B^{N} \\
C I L Q_{i j}=\frac{S L Q_{i}}{S L Q_{j}} \\
0 \leq \delta \leq 1
\end{gathered}
$$

$F L Q_{i j}=$ coeficiente de localización de Flegg del sector $i$ en relación con el sector $j$.

$C I L Q_{i j}=$ coeficiente de localización de industria cruzada del sector $i$ en relación con el sector $j$.

$S L Q_{j}=$ coeficiente de localización simple del sector $j$, es el cociente de la participación del PIB regional del sector $i$ en el PIB regional total $\left(\mathrm{PIB}^{\mathrm{R}}\right)$ y de la participación del PIB nacional del sector $i$ en el PIB nacional total $\left(\mathrm{PIB}^{\mathrm{N}}\right)$.

Supraíndices: $\mathrm{N}=$ nacional, $\mathrm{R}=$ Sinaloa

En este sentido, $\operatorname{los} a_{i j}{ }^{R}$ para el estado de Sinaloa se obtienen:

$$
\begin{aligned}
& a_{i j}^{R}=a_{i j}^{N} \text { si } F L Q_{i j}{ }^{3} 1 \\
& a_{i j}^{R}=F L Q_{i j}{ }^{*} a_{i j}^{N} \text { si } F L Q_{i j}<1
\end{aligned}
$$


De esta manera, el consumo intermedio de origen regional del sector $j$ proveniente del sector $i\left(x_{i j}{ }^{R}\right)$, el consumo intermedio total del sector $j$ de origen regional $\left(C I_{j}^{R}\right)$ y el consumo intermedio total del sector $j$ proveniente del resto de los estados $\left(C I R E_{j}\right)$ se calculan:

$$
\begin{gathered}
x_{i j}^{R}=a_{i j}{ }^{R} * P B_{j}^{R} \\
C I_{j}^{R}=\sum_{i=1}^{24} x_{i j}{ }^{R} \\
C I R E_{j}=C I T_{j}^{R}-C I_{j}^{R}
\end{gathered}
$$

El consumo intermedio importado por sector $\left(M M_{j}^{R}\right)$ del estado de Sinaloa se calculó mediante el método de ponderación regional al utilizar el PIB. Tal que:

$$
M M_{j}^{R}=M M_{j}^{N} * \frac{P I B_{j}^{R}}{P I B_{j}^{N}}
$$

\section{B.1.3.2 Valor Agregado y sus componentes}

El valor agregado bruto por sector del estado de Sinaloa proviene del INEGI. Mientras que las remuneraciones $\left(\mathrm{REM}_{\mathrm{j}}^{\mathrm{R}}\right)$ y el excedente bruto de explotación $\left(\mathrm{EXC}_{\mathrm{j}}^{\mathrm{R}}\right)$ fueron derivados al aplicar la participación que dichas variables tienen en el valor agregado por sector en el ámbito nacional:

$$
\begin{gathered}
R E M_{j}^{R}=V A B_{j}^{R} * \frac{R E M_{j}^{N}}{V A B_{j}^{N}} \\
E X C_{j}^{R}=V A B_{j}^{R} * \frac{E X C_{j}^{N}}{V A B_{j}^{N}}
\end{gathered}
$$




\section{B.1.3.3 Producto interno bruto}

Este se obtiene al sumar los impuestos a la producción netos de subsidios $\left(\mathrm{IMP}_{j}^{\mathrm{R}}\right)$ al valor agregado por sector. Los $\mathrm{IMP}_{j}^{\mathrm{R}}$ se obtienen al aplicar la tasa efectiva de impuestos sobre la producción netos de subsidios de cada sector, pagados en el ámbito nacional $\left(\mathrm{t}_{\mathrm{j}}^{\mathrm{N}}\right)$, al valor agregado del sector $j$ de Sinaloa:

$$
\begin{gathered}
I M P_{j}^{R}=t_{j}^{N * V A B_{j}^{R}} \\
P I B_{j}^{R}=V A B_{j}^{R}+I M P_{j}^{R}
\end{gathered}
$$

\section{B.1.3.4 Demanda final y sus componentes}

La demanda final total $\left(\mathrm{DF}_{\mathrm{i}}^{\mathrm{R}}\right)$ se calcula por condición de equilibrio general, iguala a la producción bruta a precios del productor, calculada por el lado de los recursos $\left(\mathrm{PB}_{i}^{\mathrm{R}}\right)$ menos la demanda intermedia $\left(\mathrm{DI}_{\mathrm{i}}^{\mathrm{R}}\right)$ :

$$
\begin{gathered}
D F_{i}^{R}=P B_{i}^{R}-D I_{i}^{R} \\
P B_{i}^{R}=P B_{j}^{R}=C I T_{j}^{R}+M_{j}^{R}+V A B_{j}^{R} \\
D I_{i}^{R}=\sum_{j=1}^{30} x_{i j}^{R}
\end{gathered}
$$

El consumo privado regional $\left(\mathrm{C}_{\mathrm{i}}^{\mathrm{R}}\right)$ de bienes provenientes del sector $i$ se obtuvo aplicando el método de ponderación regional basado en la población, tal que:

$$
C_{i}^{R}=\frac{P o b^{R}}{P o b^{N}} C_{i}^{N}
$$

donde, $\mathrm{C}_{\mathrm{i}}^{\mathrm{N}}$ es el consumo privado de bienes provenientes del sector $i$ en nivel nacional, $\operatorname{Pob}^{\mathrm{R}}$ es la población del estado de Sinaloa y $\operatorname{Pob}^{\mathrm{N}}$ es la 
población en el ámbito nacional. Se procede de igual forma para derivar el gasto del gobierno estatal en bienes y servicios $G_{i}^{R}$ :

$$
G_{i}^{R}=\frac{P o b^{R}}{P o b^{N}} G_{i}^{N}
$$

Para los fines de esta investigación no se derivaron los demás componentes de la demanda final, por lo que se engloban en la variable denominada "resto de la demanda final" $\left(\mathrm{RDF}_{\mathrm{j}}\right)$.

\section{Cuadro B3}

Matriz insumo producto del estado de Sinaloa, 2003

\begin{tabular}{|c|c|c|c|c|c|c|c|c|c|c|c|c|c|c|c|}
\hline ector & E1 & E2 & E3 & $\mathrm{E} 4$ & AE5 & AE6 & AE7 & AE8 & AE9 & AE10 & AE11 & NE12 & AE13 & AE14 & E15 \\
\hline E1 & 400 & 59 & 732 & & 0 & 34990 & 53 & 1884 & & & 1880 & & & & \\
\hline $\mathbf{E} 2$ & 52 & 841 & & & 0 & 0 & & 191] & & & 016 & 29 & & & 0 \\
\hline E3 & 11 & & 274 & & ( & & 90 & 466 & 3988 & & 23 & 667 & & & 59 \\
\hline AE4 & 14 & 3 & 28 & 47 & 1102 & 7187 & 34 & 40 & 1 & 37 & 47069 & 6199 & 9264 & 208 & \\
\hline AE5 & 822 & 27889 & 5748 & 4443 & 33596 & 41074 & 166198 & 6580 & 2974 & 27433 & 17238 & 19678 & 26290 & 5920 & 4669 \\
\hline AE6 & 3 & 7742 & 160 & 508 & 4958 & 539637 & 19641 & 429 & 44 & 548 & 3128 & 1226 & 591 & 764 & 380 \\
\hline E & 13 & 4667 & 3974 & 100 & 63 & 362 & 23 & 7047 & 77 & 3020 & 2762 & 472 & 387 & 680 & 339 \\
\hline $\mathbf{A E}$ & 9 & & 61 & & 144 & 413 & 23 & 019 & 40 & 060 & 1006 & 410 & 803 & 342 & 779 \\
\hline AE9 & 9 & 1 & 2 & 979 & 1 & 15051 & 70 & 272 & 2388 & 401 & 551 & 521 & 706 & 2281 & 7751 \\
\hline AE1 & 2586 & 0 & 24 & 25 & 660 & 4167 & 36521 & 2248 & 202 & 30905 & 10116 & 9213 & 2632 & 560 & 787 \\
\hline AE11 & 227 & 4211 & 2004 & 19061 & 40181 & 30457 & 28667 & 4480 & 791 & 5848 & 8963 & 7514 & 8988 & 9233 & 1689 \\
\hline $\mathbf{A E}$ & 44 & & & 73 & 150 & 9295 & & 174 & 53 & 17 & 122 & 4045 & 816 & 119 & 345 \\
\hline $\mathbf{A E}$ & 9 & 86 & 14 & 4981 & 478 & 8848 & & 83 & 91 & 190 & 888 & 1030 & 986 & 452 & 2401 \\
\hline AE1 & 286 & 51 & 214 & 572 & 333 & 3867 & 337 & 572 & 60 & 292 & 1623 & 473 & 373 & 148 & 186 \\
\hline AE1 & 0 & 0 & 0 & & 4 & 4157 & 11 & & 2 & & & & 4 & 602 & 831 \\
\hline $\mathbf{A E}$ & 0 & 0 & 1 & 10 & 79 & 258 & 314 & 863 & 42 & 160 & 766 & 202 & 332 & 929 & 73 \\
\hline AE1 & 9902 & 58878 & 18362 & 7446 & 28837 & 746913 & 979846 & 27536 & 18534 & 00023 & 09203 & 47066 & 79572 & 63718 & 27710 \\
\hline AE1 & 396 & 4178 & 11908 & 83 & 92795 & 231746 & 310741 & 10966 & 8407 & 50808 & 41435 & 19153 & 22396 & 300 & 13268 \\
\hline AE19 & 19 & 5 & 722 & 787 & 763 & 35862 & 850 & 1823 & 752 & 868 & 18150 & 4418 & 2832 & 399 & 2026 \\
\hline AE20 & 11847 & 9492 & 1659 & 21049 & 16699 & 24933 & 24450 & 1869 & 364 & 5095 & 14180 & 4973 & 4421 & 333 & 599 \\
\hline AE2 & & & 673 & 14022 & 10417 & 123731 & 90985 & 5562 & 1245 & 1247 & 21476 & 10758 & 748 & 4907 & 7842 \\
\hline AE22 & 5 & 17759 & 4990 & 5903 & 8254 & 84006 & 92589 & 6443 & 1284 & 12066 & 27156 & 9799 & 8768 & 18219 & 3018 \\
\hline $\mathbf{A E 2}$ & 0 & 0 & 0 & 3049 & 0 & 59 & & 0 & 0 & & 9000 & & & 176 & \\
\hline AE2 & 1 & 12 & 11 & 2697 & 11259 & 35401 & 151 & 8205 & 609 & 9732 & 23282 & 19091 & 13460 & 9843 & 2111 \\
\hline AE25 & 0 & 0 & 0 & & 1022 & 54 & 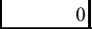 & & 0 & 34 & & & 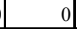 & & ? \\
\hline $\mathbf{A E}$ & 0 & 0 & 0 & & 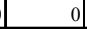 & & 0 & & 0 & & & & 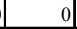 & & \\
\hline AE2 & 0 & 0 & 0 & & 0 & 5 & 12 & & 0 & 11 & & & 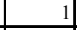 & 15 & \\
\hline AE & & 93 & 336 & 1593 & 183 & 32178 & 94 & 2699 & 275 & 221 & 4848 & 1204 & 2030 & 1684 & 2867 \\
\hline $\mathbf{A E}$ & 828 & 485 & 3377 & 4020 & 8990 & 64769 & 46618 & 2180 & 519 & 100 & 6326 & 8446 & 661 & 05 & 1712 \\
\hline AE30 & & & 2 & & 11084 & 265 & 0 & & 0 & 0 & 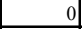 & & 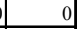 & 0 & \\
\hline CI & 2141 & 1295611 & 167679 & 113959 & 1166352 & 2300686 & \begin{tabular}{|l|l|}
6020710 \\
\end{tabular} & 100434 & 92741 & 285804 & 77105 & 176589 & 212061 & 46985 & 81446 \\
\hline CIRE & 1421637 & 969150 & 2300361 & 21806 & 2730347 & 6444056 & 7433024 & 56822 & 23948 & 234771 & 677185 & 123933 & 221302 & 179124 & 74556 \\
\hline CIT & 1883777 & 2264760 & 2468039 & 135765 & 3896700 & 8744742 & 13453734 & 157256 & 116689 & 520574 & 1154290 & 300522 & 433364 & 626109 & 156002 \\
\hline M & 781884 & 298729 & 336764 & 23051 & 727414 & \begin{tabular}{|l|l|l|}
1416319 \\
\end{tabular} & 1969226 & 150124 & 15547 & 242421 & 237890 & 65007 & 175399 & 1262470 & 121255 \\
\hline IMP & 63235 & 4531 & 64520 & 1440 & 74079 & 68983 & 39387 & 1925 & 804 & 3609 & 6490 & 4184 & 4728 & 3760 & 1688 \\
\hline VAB & 238974 & 1641883 & 5543563 & 618517 & 2954240 & 9796580 & 9849458 & 160341 & 99568 & 390917 & 467630 & 407415 & 335989 & 653387 & 191209 \\
\hline PB & 267870 & 4209904 & 8412887 & 778773 & 7652433 & 026623 & 311805 & 469645 & 32608 & 157521 & 1866300 & 777128 & 949480 & 2545727 & 4701 \\
\hline
\end{tabular}




\section{Cuadro B3}

(continúa)

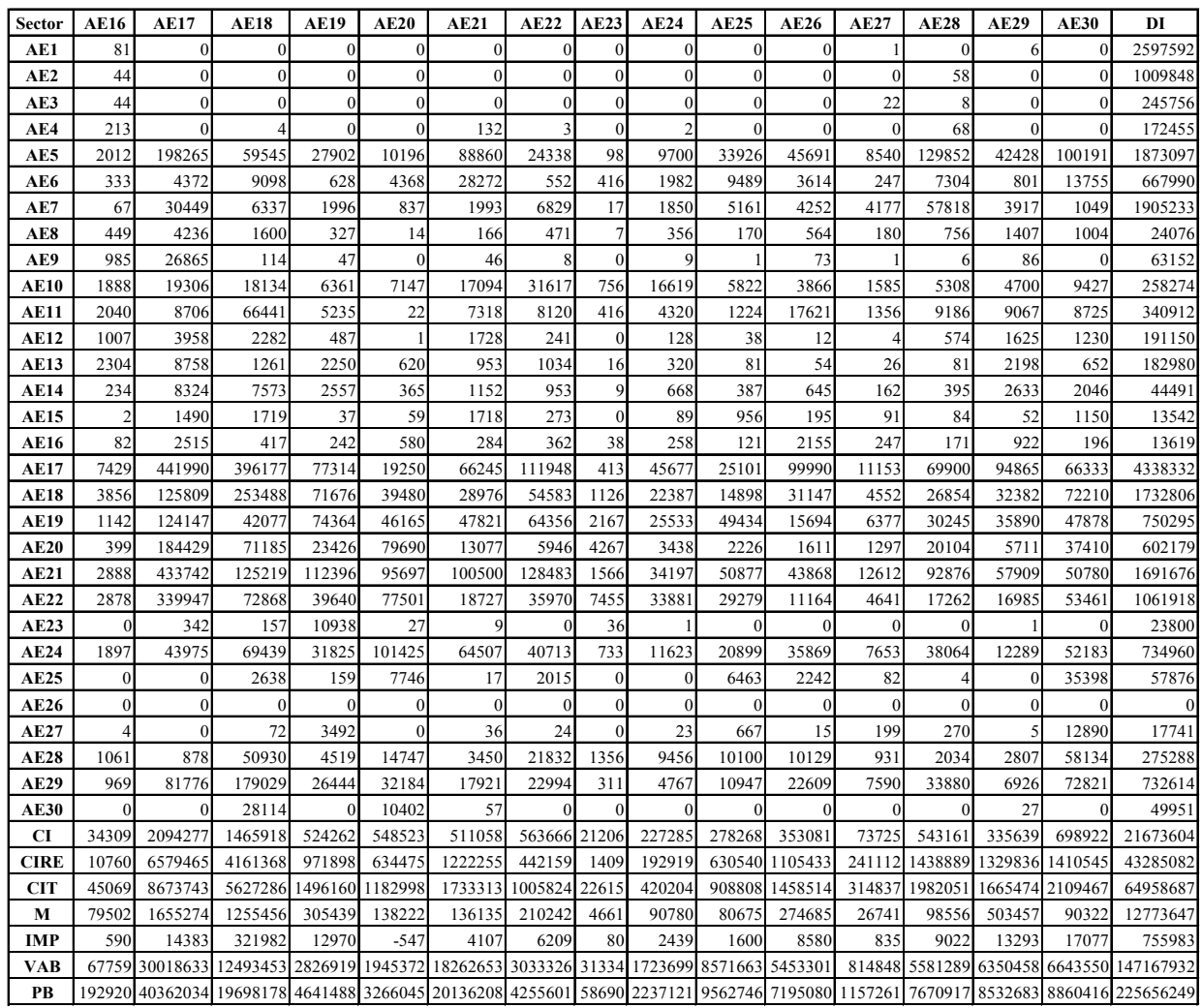

Revista de Economía - Vol. XXX - Núm. 81 


\section{Cuadro B3}

(continúa)

\begin{tabular}{|c|c|c|c|c|}
\hline Sector & $\mathrm{CP}$ & RDF & DF & PB \\
\hline AE1 & 1976753 & 8393526 & 10370279 & 12967870 \\
\hline AE2 & 1438070 & 1761986 & 3200056 & 4209904 \\
\hline AE3 & 502792 & 7664339 & 8167131 & 8412887 \\
\hline AE4 & 0 & 606318 & 606318 & 778773 \\
\hline AE5 & 2166289 & 3613047 & 5779336 & 7652433 \\
\hline AE6 & 30753 & 19327880 & 19358633 & 20026623 \\
\hline AE7 & 17936833 & 5469739 & 23406571 & 25311805 \\
\hline AE8 & 1913393 & -1467824 & 445569 & 469645 \\
\hline AE9 & 161201 & 8256 & 169457 & 232608 \\
\hline AE10 & 455962 & 443285 & 899247 & 1157521 \\
\hline AE11 & 4723572 & -3198184 & 1525388 & 1866300 \\
\hline AE12 & 777576 & -191597 & 585979 & 777128 \\
\hline AE13 & 518710 & 247789 & 766500 & 949480 \\
\hline AE14 & 3661391 & -1160156 & 2501235 & 2545727 \\
\hline AE15 & 529276 & -72664 & 456613 & 470155 \\
\hline AE16 & 329516 & -150216 & 179301 & 192920 \\
\hline AE17 & 17212304 & 18811398 & 36023702 & 40362034 \\
\hline AE18 & 13545485 & 4419886 & 17965372 & 19698178 \\
\hline AE19 & 4299683 & -408490 & 3891193 & 4641488 \\
\hline AE20 & 2405112 & 258754 & 2663866 & 3266045 \\
\hline AE21 & 17352638 & 1091894 & 18444532 & 20136208 \\
\hline AE22 & 2934064 & 259619 & 3193683 & 4255601 \\
\hline AE23 & 0 & 34890 & 34890 & 58690 \\
\hline AE24 & 544752 & 957409 & 1502162 & 2237121 \\
\hline AE25 & 3625107 & 5879763 & 9504870 & 9562746 \\
\hline AE26 & 3361209 & 3833872 & 7195080 & 7195080 \\
\hline AE27 & 904861 & 234659 & 1139520 & 1157261 \\
\hline AE28 & 6279841 & 1115788 & 7395629 & 7670917 \\
\hline AE29 & 5162508 & 2637561 & 7800069 & 8532683 \\
\hline AE30 & 109395 & 8701070 & 8810466 & 8860416 \\
\hline CI & 114859047 & 89123597 & 203982644 & 225656249 \\
\hline CIRE & 0 & 0 & 0 & 43285082 \\
\hline CIT & 114859047 & 89123597 & 203982644 & 268941331 \\
\hline $\mathbf{M}$ & 5655916 & 8898261 & 14554176 & 27327824 \\
\hline IMP & 9022592 & 0 & 9022592 & 9778574 \\
\hline VAB & 0 & 0 & 0 & 147167932 \\
\hline PB & 129537554 & 97452080 & 226989634 & 452645883 \\
\hline
\end{tabular}

Fuente: elaboración propia con base en la matriz insumo producto nacional 2003, el PIB nacional y estatal por sectores y la población estatal y nacional, INEGI. 